\title{
Normalized Solutions of Nonautonomous Kirchhoff Equations: Sub- and Super-critical Cases
}

\author{
Sitong Chen ${ }^{1}$ - Vicenţiu D. Rădulescu ${ }^{2,3}$ (D) Xianhua Tang ${ }^{1}$
}

Published online: 29 January 2020

(c) The Author(s) 2020

\section{Abstract}

In this paper, we establish the existence of normalized solutions to the following Kirchhoff-type equation

$$
\left\{\begin{array}{l}
-\left(a+b \int_{\mathbb{R}^{3}}|\nabla u|^{2} \mathrm{~d} x\right) \Delta u-\lambda u=K(x) f(u), x \in \mathbb{R}^{3} ; \\
u \in H^{1}\left(\mathbb{R}^{3}\right),
\end{array}\right.
$$

where $a, b>0, \lambda$ is unknown and appears as a Lagrange multiplier, $K \in$ $\mathcal{C}\left(\mathbb{R}^{3}, \mathbb{R}^{+}\right)$with $0<\lim _{|y| \rightarrow \infty} K(y) \leq \inf _{\mathbb{R}^{3}} K$, and $f \in \mathcal{C}(\mathbb{R}, \mathbb{R})$ satisfies general $L^{2}$-supercritical or $L^{2}$-subcritical conditions. We introduce some new analytical techniques in order to exclude the vanishing and the dichotomy cases of minimizing sequences due to the presence of the potential $K$ and the lack of the homogeneity of the nonlinearity $f$. This paper extends to the nonautonomous case previous results on prescribed $L^{2}$-norm solutions of Kirchhoff problems.

Keywords Kirchhoff problem · Normalized solution - Concentration-compactness · Indefinite potential $\cdot L^{2}$-supercritical growth $\cdot L^{2}$-subcritical growth

Mathematics Subject Classification 35J20 · 35J65

$凶$ Vicenţiu D. Rădulescu

radulescu@inf.ucv.ro

Sitong Chen

mathsitongchen@163.com

Xianhua Tang

tangxh@mail.csu.edu.cn

1 School of Mathematics and Statistics, Central South University, Changsha, Hunan 410083, People's Republic of China

2 Faculty of Applied Mathematics, AGH University of Science and Technology, al. Mickiewicza 30, 30-059 Kraków, Poland

3 Department of Mathematics, University of Craiova, A.I. Cuza No. 13, 200585 Craiova, Romania 


\section{Introduction}

This paper deals with the existence of normalized solutions to the following nonautonomous Kirchhoff-type equation:

$$
\left\{\begin{array}{l}
-\left(a+b \int_{\mathbb{R}^{3}}|\nabla u|^{2} \mathrm{~d} x\right) \Delta u-\lambda u=K(x) f(u), x \in \mathbb{R}^{3} ; \\
u \in H^{1}\left(\mathbb{R}^{3}\right),
\end{array}\right.
$$

where $a, b$ are positive real numbers, $\lambda$ is unknown and will appear as a Lagrange multiplier, $K \in \mathcal{C}\left(\mathbb{R}^{3}, \mathbb{R}^{+}\right)$and $f \in \mathcal{C}(\mathbb{R}, \mathbb{R})$. This equation is related to the stationary analogue of the Kirchhoff equation

$$
u_{t t}-\left(a+b \int_{\mathbb{R}^{3}}|\nabla u|^{2} \mathrm{~d} x\right) \Delta u=g(x, t)
$$

The Kirchhoff equation has been introduced for the first time in 1883 by Kirchhoff [16] in dimension 1, without forcing term and with Dirichlet boundary conditions, in order to describe the transversal free vibrations of a clamped string in which the dependence of the tension on the deformation cannot be neglected. This is a quasilinear partial differential equation, namely the nonlinear part of the equation contains as many derivatives as the linear differential operator. The Kirchhoff equation is an extension of the classical D'Alembert wave equation for free vibrations of elastic strings. Kirchhoff's model takes into account the changes in length of the string produced by transverse vibrations. We refer to $[1,5,6,12]$ for the physical background on Kirchhoff's model.

From the mathematical point of view, problem (1.1) is nonlocal since the appearance of the term $\int_{\mathbb{R}^{3}}|\nabla u|^{2} \mathrm{~d} x$ indicates that (1.1) is not a pointwise identity. This kind of problem has been paid much attention after the pioneering work of Lions [19], in which an abstract functional analysis framework was introduced.

If $\lambda \in \mathbb{R}$ is a fixed parameter or even in the presence of an additional external and fixed potential $V(x)$, the existence of solutions of problem (1.1) has been intensively studied during the last decade; see, for example, [2,7,13,17,18,20-22,24,32] and the references therein. In this case, solutions can be obtained as critical points of the corresponding energy functional, but without any information on the $L^{2}$-norm of the solutions.

Nowadays, since physicists are interested in normalized solutions, mathematical researchers began to focus on solutions having a prescribed $L^{2}$-norm, that is, solutions which satisfy $\|u\|_{2}^{2}=c>0$ for a priori given $c$. To the best of our knowledge, the study of solutions with prescribed norm was initiated by Jeanjean [14] in the framework of semilinear elliptic equations. We also refer to Bellazzini et al. [4] and Cingolani and Jeanjean [11] for normalized solutions of the Schrödinger-Poisson system. In the present paper, we are interested in the existence of solutions with $L^{2}$-prescribed norm and their qualitative properties in the framework of nonlocal Kirchhoff problems. Our analysis includes both the $L^{2}$-supercritical case and the $L^{2}$-subcritical growth. 
Solutions of problem (1.1) with $\|u\|_{2}^{2}=c>0$ can be obtained by looking for critical points of the following functional

$$
I(u)=\frac{a}{2} \int_{\mathbb{R}^{3}}|\nabla u|^{2} \mathrm{~d} x+\frac{b}{4}\left(\int_{\mathbb{R}^{3}}|\nabla u|^{2} \mathrm{~d} x\right)^{2}-\int_{\mathbb{R}^{3}} K(x) F(u) \mathrm{d} x
$$

on the manifold

$$
\mathcal{S}_{c}=\left\{u \in H^{1}\left(\mathbb{R}^{3}\right):\|u\|_{2}^{2}=c\right\},
$$

where $F(u)=\int_{0}^{u} f(t) \mathrm{d} t$. In this case, the parameter $\lambda \in \mathbb{R}$ cannot be fixed but instead it appears as a Lagrange multiplier, and each critical point $u_{c} \in \mathcal{S}_{c}$ of $\left.I\right|_{\mathcal{S}_{c}}$, corresponds to a Lagrange multiplier $\lambda_{c} \in \mathbb{R}$ such that $\left(u_{c}, \lambda_{c}\right)$ solves (weakly) problem (1.1). In particular, if $u_{c} \in \mathcal{S}_{c}$ is a solution of the constrained minimization problem

$$
\sigma(c):=\inf _{u \in \mathcal{S}_{c}} I(u)
$$

then there exists $\lambda_{c} \in \mathbb{R}$ such that $I^{\prime}\left(u_{c}\right)=\lambda_{c} u_{c}$, that is, $\left(u_{c}, \lambda_{c}\right)$ is a solution of problem (1.1).

To the best of our knowledge, solutions of problem (1.1) having a prescribed $L^{2}$ norm have been studied only if $K(x) \equiv 1$; see, e.g., [28-31,33]. Let us introduce and review the few known results in this respect. Ye [29] studied the existence and non-existence of normalized solutions to the special form of (1.1):

$$
\left\{\begin{array}{l}
-\left(a+b \int_{\mathbb{R}^{3}}|\nabla u|^{2} \mathrm{~d} x\right) \Delta u-\lambda u=|u|^{p-2} u, x \in \mathbb{R}^{3} \\
u \in H^{1}\left(\mathbb{R}^{3}\right)
\end{array}\right.
$$

for $p \in(2,6)$, and showed that $p=\frac{14}{3}$ is a $L^{2}$-critical exponent for problem (1.6), that is, for any given $c>0$,

$$
\begin{cases}\sigma(c) \in(-\infty, 0], & \text { if } p \in\left(2, \frac{14}{3}\right) \\ \sigma(c)=-\infty, & \text { if } p \in\left(\frac{14}{3}, 6\right)\end{cases}
$$

More precisely, for any $p \in\left(2, \frac{14}{3}\right)$, Ye [29] obtained the sharp existence of global constraint minimizers for (1.6) by solving the minimization problem (1.5). If $p \in$ $\left(\frac{10}{3}, \frac{14}{3}\right)$, Ye [29] found a local minimizer which is also a critical point of $\left.I\right|_{\mathcal{S}_{c}}$ by constructing a geometry of local minima for $I$. For the case $p \in\left(\frac{14}{3}, 6\right)$, since the minimization problem (1.5) is not available due to (1.7), to look for a critical point of $\left.I\right|_{\mathcal{S}_{c}}$, Ye [29] took a minimum on a suitable submanifold

$$
\mathcal{M}_{c}:=\left\{u \in \mathcal{S}_{c}: J(u):=\left.\frac{\mathrm{d}}{\mathrm{d} t} I\left(u^{t}\right)\right|_{t=1}=0\right\},
$$

and showed that $\mathcal{M}_{c}$ is a natural constraint of $\left.I\right|_{\mathcal{S}_{c}}$ by using the Lagrange multiplier method, where

$$
u^{t}(x):=t^{3 / 2} u(t x), \quad \forall t>0, u \in H^{1}\left(\mathbb{R}^{3}\right),
$$


and $u^{t} \in \mathcal{S}_{c}$ for all $t>0$ if $u \in \mathcal{S}_{c}$. Note that this method relies heavily on the powertype nonlinearity $f(u)=|u|^{p-2} u$ with $p \in\left(\frac{14}{3}, 6\right)$. Recently, in [28], Xie and Chen generalized this special case to general nonlinearities $f$ satisfying $\lim _{|t| \rightarrow \infty} \frac{F(t)}{|t|^{14 / 3}}=$ $+\infty$. Using some ideas developed in [24,29], Xie and Chen [28] proved the existence of normalized solutions for problem (1.1) with $K(x) \equiv 1$ under additional growth and monotonicity conditions. For the $L^{2}$-critical case, Ye [30] proved that problem (1.6) with $p=\frac{14}{3}$ has a solution $\left(u_{c}, \lambda_{c}\right)$ which satisfies $\left\|u_{c}\right\|_{2}^{2}=c>c^{*}$ for some $c^{*}>0$. We also recall that Ye [31] also analyzed the concentration behavior of solutions. If $p \in\left(2, \frac{14}{3}\right)$, by using a different method, Zeng and Zhang [33] proved the existence and uniqueness of normalized solutions for problem (1.6). Additionally, it was considered in $[29-31,33]$ the existence of normalized solutions for one-dimensional and twodimensional autonomous Kirchhoff type equations with power-type nonlinearity.

Let us also emphasize that all of the strategies used in [28-31,33] only work for autonomous problems, and fail to adapt directly to problem (1.1) with non-constant potential $K(x)$. To the best of our knowledge, there are no results dealing with the non-autonomous abstract setting. The main purpose of this paper is to extend and complement the corresponding existence results in [29] to problem (1.1) in the presence of the variable potential $K(x)$.

\section{Main Results}

Motivated by the above works, we first consider the $L^{2}$-supercritical case, and establish the existence of a critical point of $I$ on $\mathcal{S}_{c}$ by considering the constrained minimization problem

$$
m(c):=\inf _{u \in \mathcal{M}_{c}} I(u)
$$

where the definition of $\mathcal{M}_{c}$ is given by (1.8). To this end, we introduce the following assumptions:

(F1) $f \in \mathcal{C}(\mathbb{R}, \mathbb{R})$, there exists $\mu \in\left(\frac{14}{3}, 6\right)$ such that $0 \leq f(t) t \leq \mu F(t)$ for all $t \in \mathbb{R}$, and meas $\{t \in \mathbb{R}: \mu F(t)-f(t) t=0\}=0 ;$

(F2) there exists $\theta \in\left(2, \frac{14}{3}\right)$ such that $\lim _{|t| \rightarrow 0} \frac{F(t)}{|t|^{\theta}}=0$ and $\lim _{|t| \rightarrow \infty} \frac{F(t)}{|t|^{14 / 3}}=+\infty$;

(F3) the mapping $t \mapsto[f(t) t-\theta F(t)] /|t|^{11 / 3} t$ is nondecreasing on $(-\infty, 0)$ and $(0,+\infty)$

(K1) $K \in \mathcal{C}\left(\mathbb{R}^{3}, \mathbb{R}^{+}\right)$and $0<K_{\infty}:=\lim _{|y| \rightarrow \infty} K(y) \leq K(x)$ for all $x \in \mathbb{R}^{3}$;

(K2) $K \in \mathcal{C}^{1}\left(\mathbb{R}^{3}, \mathbb{R}^{+}\right),(6-\mu) K(x)+2 \nabla K(x) \cdot x \geq 0$ for all $x \in \mathbb{R}^{3}$, and $3(\theta-2) K(t x)-2 \nabla K(t x) \cdot(t x)$ is nonincreasing on $t \in(0, \infty)$ for every $x \in \mathbb{R}^{3}$.

Our first result establishes the following qualitative property.

Theorem 2.1 Assume that (K1), (K2) and (F1)-(F3) hold. Then for any $c>0$, problem (1.1) has a couple of solution $\left(\bar{u}_{c}, \lambda_{c}\right) \in \mathcal{S}_{c} \times \mathbb{R}^{-}$such that 


$$
I\left(\bar{u}_{c}\right)=\inf _{u \in \mathcal{M}_{c}} I(u)=\inf _{u \in \mathcal{S}_{c}} \max _{t>0} I\left(u^{t}\right)>0,
$$

where the definitions of $\mathcal{M}_{c}$ and $u^{t}$ are given by (1.8) and (1.9).

Next, in the $L^{2}$-subcritical case, we find a global minimizer and a local minimizer of $I$ which are critical points of $\left.I\right|_{\mathcal{S}_{c}}$ by solving the minimization problem (1.5) and constructing a geometry of local minima for $I$ (see Lemma 4.6), respectively. To this end, in addition to (K1), we introduce the following assumptions:

(F4) $f \in \mathcal{C}(\mathbb{R}, \mathbb{R}), f(t)=o(t)$ as $t \rightarrow 0$ and there exist constants $\mathcal{C}>0$ and $p \in\left(\frac{10}{3}, \frac{14}{3}\right)$ such that $|f(t)| \leq \mathcal{C}\left(1+|t|^{p-1}\right)$;

(F5) there exists $\mu_{0} \in\left(2, \frac{14}{3}\right)$ such that $f(t) t \geq \mu_{0} F(t)>0$ for all $t \in \mathbb{R} \backslash\{0\}$;

(F6) there exists $q_{0} \in\left(2, \frac{10}{3}\right)$ such that $\lim _{|t| \rightarrow 0} \frac{F(t)}{|t|^{q_{0}}}>0$;

(F6') $\lim _{|t| \rightarrow 0} \frac{F(t)}{|t|^{10 / 3}}=0$;

(K3) the mapping $t \mapsto t^{\left(\mu_{0}-2\right) / 2} K(t x)$ is nondecreasing on $(0, \infty)$ for every $x \in \mathbb{R}^{3}$.

Let

$$
c_{*}:=\inf \{c \in(0,+\infty), \sigma(c)<0\} .
$$

We have the following statement.

Theorem 2.2 Assume that $K$ and $f$ satisfy (K1), (K3), (F4) and (F5).

(i) If (F6) holds, then $c_{*}=0$ and I admits a critical point $u_{c}$ on $\mathcal{S}_{c}$, which is a negative global minimum of I when $c>0$.

(ii) If $\left(F 6^{\prime}\right)$ holds, then $c_{*}>0$, and there exists $c_{0} \in\left(0, c_{*}\right)$ such that I admits a critical point $u_{c}$ on $\mathcal{S}_{c}$ which is a local minimum of I when $c \in\left(c_{0}, c_{*}\right)$, but $u_{c}$ is a global minimum of I when $c \in\left[c_{*},+\infty\right)$. In particular,

$$
I\left(u_{c}\right)\left\{\begin{array}{l}
>0, \text { if } c \in\left(c_{0}, c_{*}\right) ; \\
=0, \text { if } c=c_{*} ; \\
<0, \text { if } c \in\left[c_{*}, \infty\right) .
\end{array}\right.
$$

Moreover, for the above critical point $u_{c}$, there is a Lagrange multiplier $\lambda_{c} \in \mathbb{R}$ such that $\left(u_{c}, \lambda_{c}\right)$ is a solution of problem (1.1).

Remark 2.3 Theorems 2.1 and 2.2 make a substantial improvement and extension to the main results in [29]. In particular, if $K(x) \equiv 1$, the conclusion of Theorem 2.1 holds under hypotheses (F1)-(F3) with $\theta=2$, and it reduces to the result of [28, Theorem 1.1].

Compared with the previous works, we have to overcome the essential difficulties that the variable potential $K(x)$ gives rise when searching for normalized solutions of problem (1.1). These difficulties enforce the implementation of new ideas and techniques for the proof of Theorems 2.1 and 2.2. Let us point them out in more detail.

For the $L^{2}$-supercritical case, some useful remarks are stated in what follows. 
- When $K(x) \equiv K_{\infty}$, Ye [29] showed that

$$
c \mapsto m(c) \text { is strictly decreasing on }(0,+\infty)
$$

by using the translation invariance of $I$ and the homogeneity of $f$. Then Ye can exclude the vanishing and the dichotomy cases of the minimizing sequence $\left\{u_{n}\right\}$ for $m(c)=\inf _{u \in \mathcal{M}_{c}} I(u)$ in applying the concentration-compactness principle. However, the approach used in [29] is valid only for autonomous equations and it does not work any more for (1.1) with $K \neq$ constant and more general $f$, see Remark 3.10 for more details. Unlike [29], by establishing some new inequalities, we prove that $m(c)$ is nonincreasing and $m(c)>m(\tilde{c})$ for any $\tilde{c}>c$ provided $m(c)$ is attained. To bypass the difficulty caused by the lack of compactness of Sobolev embedding $H^{1}\left(\mathbb{R}^{3}\right) \hookrightarrow L^{s}\left(\mathbb{R}^{3}\right)$ for $2 \leq s<6$, we compare the constrained minimum $m(c)$ with the one of the "limit equation" (that is, problem (1.1) with $\left.K(x)=K_{\infty}\right)$, and by using some subtle analysis we prove that $u_{n} \rightarrow \bar{u}$ in $H^{1}\left(\mathbb{R}^{3}\right.$ ) (after a translation and extraction of a subsequence if necessary) as long as $m(c)<m^{\infty}(c)$, and $\bar{u} \in \mathcal{S}(c)$ is a minimizer of $m(c)$, where the definition of $m^{\infty}(c)$ is given by (2.10).

- To verify that $\mathcal{M}_{c}$ is a natural constraint on $\mathcal{S}_{c}$, we use a combination of the deformation lemma, some new inequalities and an intermediary theorem for continuous functions, other than the mountain pass theorem on $\mathcal{S}_{c}$ and the Lagrange multiplier method used in [28,29], respectively.

For the $L^{2}$-subcritical case, some remarks are as follows.

- The key step to prove that $\sigma(c)=\inf _{\mathcal{S}_{c}} I$ is achieved is to obtain the subadditivity inequality

$$
\sigma(c)<\sigma(\alpha)+\sigma(c-\alpha), \quad \forall 0<\alpha<c .
$$

To this end, Ye in [29] used the scaling $t \mapsto u\left(t^{-2 / 3} x\right)$. But this kind of scaling is not suitable in our case, excepting the case when $K(x)$ is a positive constant. Instead, we present another scaling $t \mapsto t^{1 / 2} u(x / t)$, and we succeed to prove that (2.5) still holds under assumptions of Theorem 2.2.

- Compared with [29], it is more complicated to find a local minimizer of $I$ on $\mathcal{S}_{c}$ which is a critical point of $\left.I\right|_{\mathcal{S}_{c}}$ because $K(x)$ is variable and $f$ is nonhomogeneous. For this purpose, we make some improvements of the method used in [29] and employ some subtle analysis in the proofs.

When $K \in \mathcal{C}\left(\mathbb{R}^{3}, \mathbb{R}^{+}\right)$is bounded, $f$ satisfies (F1) and (F2) (or (F4)), we deduce by a standard argument that $I \in \mathcal{C}^{1}\left(H^{1}\left(\mathbb{R}^{3}\right), \mathbb{R}\right)$. Let us define the "limit equation" associated to problem (1.1) by

$$
\left\{\begin{array}{l}
-\left(a+b \int_{\mathbb{R}^{3}}|\nabla u|^{2} \mathrm{~d} x\right) \Delta u-\lambda u=K_{\infty} f(u), x \in \mathbb{R}^{3} \\
u \in H^{1}\left(\mathbb{R}^{3}\right) .
\end{array}\right.
$$

Corresponding to (1.3), (1.5), (1.8) and (2.1), we define

$$
I^{\infty}(u)=\frac{a}{2} \int_{\mathbb{R}^{3}}|\nabla u|^{2} \mathrm{~d} x+\frac{b}{4}\left(\int_{\mathbb{R}^{3}}|\nabla u|^{2} \mathrm{~d} x\right)^{2}-\int_{\mathbb{R}^{3}} K_{\infty} F(u) \mathrm{d} x,
$$




$$
\begin{aligned}
\sigma^{\infty}(c) & =\inf _{u \in \mathcal{S}_{c}} I^{\infty}(u), \\
\mathcal{M}_{c}^{\infty} & =\left\{u \in \mathcal{S}_{c}: J^{\infty}(u):=\left.\frac{\mathrm{d}}{\mathrm{d} t} I^{\infty}\left(u^{t}\right)\right|_{t=1}=0\right\}
\end{aligned}
$$

and

$$
m^{\infty}(c)=\inf _{u \in \mathcal{M}_{c}^{\infty}} I^{\infty}(u)
$$

Remark that all above conclusions on problem (1.1) in this paper are also true for the limit equation (2.6), since $K(x) \equiv K_{\infty}$ satisfies (K1)-(K3).

Let $a>0$ and $b>0$ be fixed. Throughout this paper we make use of the following notations:

- $H^{1}\left(\mathbb{R}^{3}\right)$ denotes the usual Sobolev space equipped with the inner product and norm

$$
(u, v)=\int_{\mathbb{R}^{3}}(\nabla u \cdot \nabla v+u v) \mathrm{d} x, \quad\|u\|=(u, u)^{1 / 2}, \quad \forall u, v \in H^{1}\left(\mathbb{R}^{3}\right)
$$

- $L^{s}\left(\mathbb{R}^{3}\right)(1 \leq s<\infty)$ denotes the Lebesgue space with the norm $\|u\|_{s}=$ $\left(\int_{\mathbb{R}^{3}}|u|^{s} \mathrm{~d} x\right)^{1 / s}$;

- For any $u \in H^{1}\left(\mathbb{R}^{3}\right), u^{t}(x):=t^{3 / 2} u(t x)$ and $u_{t}(x):=t^{1 / 2} u(x / t)$;

- For any $x \in \mathbb{R}^{3}$ and $r>0, B_{r}(x):=\left\{y \in \mathbb{R}^{3}:|y-x|<r\right\}$;

- $S=\inf _{u \in \mathcal{D}^{1,2}\left(\mathbb{R}^{3}\right) \backslash\{0\}}\|\nabla u\|_{2}^{2} /\|u\|_{6}^{2}$;

- $C_{1}, C_{2}, \ldots$ denote positive constants possibly different in different places.

\section{First Existence Result}

In this section, we give the proof of Theorem 2.1 .

Lemma 3.1 Assume that hypotheses (K1) and (K2) hold. Then

$$
\begin{aligned}
& h_{0}(x, t):=t^{\frac{3(\theta-2)}{2}}\left[K\left(t^{-1} x\right)-K(x)\right] \\
& \quad-\frac{2\left(1-t^{\frac{3(\theta-2)}{2}}\right)}{3(\theta-2)} \nabla K(x) \cdot x \geq 0, \quad \forall x \in \mathbb{R}^{3}, t>0, \\
& \quad t \mapsto K(t x) \text { is nonincreasing on }(0, \infty) \text { for every } x \in \mathbb{R}^{3},
\end{aligned}
$$

and

$$
|\nabla K(x) \cdot x| \rightarrow 0 \text { as }|x| \rightarrow \infty
$$


Proof For any $x \in \mathbb{R}^{3}$, by (K2), we have

$$
\begin{aligned}
& \frac{\mathrm{d}}{\mathrm{d} t} h_{0}(x, t)= \frac{t^{\frac{3(\theta-2)}{2}-1}}{2}\left[3(\theta-2) K\left(t^{-1} x\right)-2 \nabla K\left(t^{-1} x\right) \cdot\left(t^{-1} x\right)\right. \\
&-3(\theta-2) K(x)+2 \nabla K(x) \cdot x] \\
&\left\{\begin{array}{l}
\geq 0, t \geq 1 \\
\leq 0,0<t<1,
\end{array}\right.
\end{aligned}
$$

which implies that $h_{0}(x, t) \geq h_{0}(x, 1)=0$ for all $x \in \mathbb{R}^{3}$ and $t>0$, hence relation (3.1) holds. By (3.1) and the continuity of $h_{0}(x, \cdot)$, we have

$$
\lim _{t \rightarrow 0} h_{0}(x, t)=-\frac{2}{3(\theta-2)} \nabla K(x) \cdot x \geq 0, \quad \forall x \in \mathbb{R}^{3}
$$

which leads to (3.2). Since $h_{0}(x, 2) \geq 0$ for all $x \in \mathbb{R}^{3}$, we have

$$
0 \leq-\nabla K(x) \cdot x \leq \frac{2^{\frac{3 \theta-8}{2}} 3(\theta-2)[K(x / 2)-K(x)]}{2^{\frac{3(\theta-2)}{2}}-1}, \quad \forall x \in \mathbb{R}^{3}
$$

Thus, relation (3.3) holds by letting $|x| \rightarrow \infty$ in the above inequality.

Lemma 3.2 Assume that hypotheses (F1)-(F3) hold. Then

$$
\begin{aligned}
h_{1}(t, \tau) & :=\frac{2\left(1-t^{7-3 \theta / 2}\right)}{14-3 \theta}[f(\tau) \tau-\theta F(\tau)]-\frac{2}{3} F(\tau)+\frac{2}{3} t^{-3 \theta / 2} F\left(t^{3 / 2} \tau\right) \\
& \geq 0, \quad \forall t>0, \quad \tau \in \mathbb{R}
\end{aligned}
$$

and

$$
\frac{F(t)}{|t|^{11 / 3} t} \text { is nondecreasing on both }(-\infty, 0) \text { and }(0,+\infty) \text {. }
$$

Proof For any $\tau \in \mathbb{R}$, by (F3), we have

$$
\begin{aligned}
\frac{\mathrm{d}}{\mathrm{d} t} h_{1}(t, \tau) & =t^{6-3 \theta / 2}|\tau|^{14 / 3}\left[\frac{f\left(t^{3 / 2} \tau\right) t^{3 / 2} \tau-\theta F\left(t^{3 / 2} \tau\right)}{\left|t^{3 / 2} \tau\right|^{14 / 3}}-\frac{f(\tau) \tau-\theta F(\tau)}{|\tau|^{14 / 3}}\right] \\
& \left\{\begin{array}{l}
\geq 0, t \geq 1 \\
\leq 0,0<t<1,
\end{array}\right.
\end{aligned}
$$

which implies that $h_{1}(t, \tau) \geq h_{1}(1, \tau)=0$ for all $t>0$ and $\tau \in \mathbb{R}$, that is, inequality (3.5) holds. By (F3) and (3.5), we obtain

$$
h_{1}(0, \tau):=\lim _{|t| \rightarrow 0} h(t, \tau)=\frac{2}{14-3 \theta}\left[f(\tau) \tau-\frac{14}{3} F(\tau)\right] \geq 0, \quad \forall \tau \in \mathbb{R} .
$$


From (3.7), we derive

$$
\frac{\mathrm{d}}{\mathrm{d} t} \frac{F(t)}{|t|^{11 / 3} t}=\frac{3}{2|t|^{7}}\left[f(t) t-\frac{14}{3} F(t)\right] \geq 0 .
$$

This shows that property (3.6) holds.

Lemma 3.3 Assume that hypotheses (K1), (K2) and (F1)-(F3) hold. Then

$$
\begin{aligned}
h_{2}(x, t, \tau):= & t^{-3} K\left(t^{-1} x\right) F\left(t^{3 / 2} \tau\right)-K(x) F(\tau)+\frac{3\left(1-t^{4}\right)}{8} K(x)[f(\tau) \tau-2 F(\tau)] \\
& -\frac{1-t^{4}}{4} \nabla K(x) \cdot x F(\tau) \\
\geq & 0, \quad \forall x \in \mathbb{R}^{3}, t>0, \tau \in \mathbb{R} .
\end{aligned}
$$

Proof For any $x \in \mathbb{R}^{3}$ and $\tau \in \mathbb{R}$, by (K1), (K2), (F1), (F3), (3.2) and (3.6), we have

$$
\begin{aligned}
\frac{\mathrm{d}}{\mathrm{d} t} h_{2}(x, t, \tau)= & -\frac{1}{t^{4}} \nabla K\left(t^{-1} x\right) \cdot\left(t^{-1} x\right) F\left(t^{3 / 2} \tau\right)+\frac{3}{2 t^{4}} K\left(t^{-1} x\right)\left[f\left(t^{3 / 2} \tau\right) t^{3 / 2} \tau-2 F\left(t^{3 / 2} \tau\right)\right] \\
& -\frac{3 t^{3}}{2} K(x)[f(\tau) \tau-2 F(\tau)]+t^{3} \nabla K(x) \cdot x F(\tau) \\
= & \frac{3}{2 t^{4}} K\left(t^{-1} x\right)\left[f\left(t^{3 / 2} \tau\right) t^{3 / 2} \tau-\theta F\left(t^{3 / 2} \tau\right)\right] \\
& +\frac{1}{2 t^{4}}\left[3(\theta-2) K\left(t^{-1} x\right)-2 \nabla K\left(t^{-1} x\right) \cdot\left(t^{-1} x\right)\right] F\left(t^{3 / 2} \tau\right) \\
& -\frac{3 t^{3}}{2} K(x)[f(\tau) \tau-\theta F(\tau)]-\frac{t^{3}}{2}[3(\theta-2) K(x)-2 \nabla K(x) \cdot x] F(\tau) \\
= & \frac{t^{3}|\tau|^{7}}{2}\left\{3 K\left(t^{-1} x\right) \frac{f\left(t^{3 / 2} \tau\right) t^{3 / 2} \tau-\theta F\left(t^{3 / 2} \tau\right)}{\left|t^{3 / 2} \tau\right|^{14 / 3}}-3 K(x) \frac{f(\tau) \tau-\theta F(\tau)}{|\tau|^{14 / 3}}\right. \\
& +\left[3(\theta-2) K\left(t^{-1} x\right)-2 \nabla K\left(t^{-1} x\right) \cdot\left(t^{-1} x\right)\right] \frac{F\left(t^{3 / 2} \tau\right)}{\left|t^{3 / 2} \tau\right|^{14 / 3}} \\
& \left.-[3(\theta-2) K(x)-2 \nabla K(x) \cdot x] \frac{F(\tau)}{|\tau|^{14 / 3}}\right\} \\
& \left\{\begin{array}{l}
\geq 0, t \geq 1, \\
\leq
\end{array}\right\}, 0<t<1 .
\end{aligned}
$$

It follows that $h_{2}(x, t, \tau) \geq h_{2}(x, 1, \tau)=0$ for all $x \in \mathbb{R}^{3}, t>0$ and $\tau \in \mathbb{R}$, hence relation (3.8) holds.

By the scaling (1.9), we have

$$
I\left(u^{t}\right)=\frac{a t^{2}}{2} \int_{\mathbb{R}^{3}}|\nabla u|^{2} \mathrm{~d} x+\frac{b t^{4}}{4}\left(\int_{\mathbb{R}^{3}}|\nabla u|^{2} \mathrm{~d} x\right)^{2}-t^{-3} \int_{\mathbb{R}^{3}} K\left(t^{-1} x\right) F\left(t^{3 / 2} u\right) \mathrm{d} x
$$


and

$$
I^{\infty}\left(u^{t}\right)=\frac{a t^{2}}{2} \int_{\mathbb{R}^{3}}|\nabla u|^{2} \mathrm{~d} x+\frac{b t^{4}}{4}\left(\int_{\mathbb{R}^{3}}|\nabla u|^{2} \mathrm{~d} x\right)^{2}-t^{-3} \int_{\mathbb{R}^{3}} K_{\infty} F\left(t^{3 / 2} u\right) \mathrm{d} x .
$$

Noting that $J(u)=\left.\frac{\mathrm{d}}{\mathrm{d} t} I\left(u^{t}\right)\right|_{t=1}$ and $J^{\infty}(u):=\left.\frac{\mathrm{d}}{\mathrm{d} t} I^{\infty}\left(u^{t}\right)\right|_{t=1}$, it follows from (3.9) and (3.10) that

$$
\begin{aligned}
J(u)= & a\|\nabla u\|_{2}^{2}+b\|\nabla u\|_{2}^{4}-\frac{3}{2} \int_{\mathbb{R}^{3}} K(x)[f(u) u-2 F(u)] \mathrm{d} x \\
& +\int_{\mathbb{R}^{3}} \nabla K(x) \cdot x F(u) \mathrm{d} x
\end{aligned}
$$

and

$$
J^{\infty}(u)=a\|\nabla u\|_{2}^{2}+b\|\nabla u\|_{2}^{4}-\frac{3}{2} \int_{\mathbb{R}^{3}} K_{\infty}[f(u) u-2 F(u)] \mathrm{d} x .
$$

Inspired by $[9,10]$, we prove the following lemma.

Lemma 3.4 Assume that hypotheses (K1), (K2) and (F1)-(F3) hold. Then

$$
I(u) \geq I\left(u^{t}\right)+\frac{1-t^{4}}{4} J(u)+\frac{a\left(1-t^{2}\right)^{2}}{4}\|\nabla u\|_{2}^{2}, \quad \forall u \in H^{1}\left(\mathbb{R}^{3}\right), t>0 .
$$

Proof By (1.3), (3.5), (3.6), (3.8), (3.9) and (3.11), we have

$$
\begin{aligned}
I(u)-I\left(u^{t}\right)= & \frac{a\left(1-t^{2}\right)}{2}\|\nabla u\|_{2}^{2}+\frac{b\left(1-t^{4}\right)}{4}\|\nabla u\|_{2}^{4} \\
& +\int_{\mathbb{R}^{3}}\left[t^{-3} K\left(t^{-1} x\right) F\left(t^{3 / 2} u\right)-K(x) F(u)\right] \mathrm{d} x \\
= & \frac{1-t^{4}}{4}\left\{a\|\nabla u\|_{2}^{2}+b\|\nabla u\|_{2}^{4}-\frac{3}{2} \int_{\mathbb{R}^{3}} K(x)[f(u) u-2 F(u)] \mathrm{d} x\right. \\
& \left.+\int_{\mathbb{R}^{3}} \nabla K(x) \cdot x F(u) \mathrm{d} x\right\}+\frac{a\left(1-t^{2}\right)^{2}}{4}\|\nabla u\|_{2}^{2} \\
& +\int_{\mathbb{R}^{3}}\left\{t^{-3} K\left(t^{-1} x\right) F\left(t^{3 / 2} u\right)-K(x) F(u)\right. \\
& +\frac{3\left(1-t^{4}\right)}{8} K(x)[f(u) u-2 F(u)] \\
& \left.-\frac{1-t^{4}}{4} \nabla K(x) \cdot x F(u)\right\} \mathrm{d} x \\
\geq & \frac{1-t^{4}}{4} J(u)+\frac{a\left(1-t^{2}\right)^{2}}{4}\|\nabla u\|_{2}^{2}, \quad \forall u \in H^{1}\left(\mathbb{R}^{3}\right), \quad t>0 .(3.14)
\end{aligned}
$$

This shows that (3.13) holds. 
From Lemma 3.4, we have the following corollary. In what follows, the definitions of $\mathcal{M}_{c}$ and $u^{t}$ are given by (1.8) and (1.9).

Corollary 3.5 Assume that hypotheses (K1), (K2) and (F1)-(F3) hold. Then

$$
I(u)=\max _{t>0} I\left(u^{t}\right), \quad \forall u \in \mathcal{M}_{c} .
$$

Lemma 3.6 Assume that hypotheses (K1), (K2) and (F1)-(F3) hold. Then for any $u \in H^{1}\left(\mathbb{R}^{3}\right) \backslash\{0\}$, there exists a unique $t_{u}>0$ such that $u^{t_{u}} \in \mathcal{M}_{c}$.

Proof Let $u \in H^{1}\left(\mathbb{R}^{3}\right) \backslash\{0\}$ be fixed and define a function $\zeta(t):=I\left(u^{t}\right)$ on $(0, \infty)$. Clearly, by (3.9) and (3.11), we have

$$
\begin{aligned}
\zeta^{\prime}(t)=0 \Leftrightarrow & a t\|\nabla u\|_{2}^{2}+b t^{3}\|\nabla u\|_{2}^{4} \\
& -\frac{3}{2 t^{4}} \int_{\mathbb{R}^{3}} K\left(t^{-1} x\right)\left[f\left(t^{3 / 2} u\right) t^{3 / 2} u-2 F\left(t^{3 / 2} u\right)\right] \mathrm{d} x \\
& +\frac{1}{t^{4}} \int_{\mathbb{R}^{3}} \nabla K\left(t^{-1} x\right) \cdot\left(t^{-1} x\right) F\left(t^{3 / 2} u\right) \mathrm{d} x=0 \\
& \Leftrightarrow \frac{1}{t} J\left(u^{t}\right)=0 \Leftrightarrow u^{t} \in \mathcal{M}_{c} .
\end{aligned}
$$

Note that (3.2) and (3.6) lead to

$$
K\left(t^{-1} x\right) F\left(t^{3 / 2} \tau\right) \leq t^{7} K(x) F(\tau), \quad \forall x \in \mathbb{R}^{3}, t \in(0,1), \tau \in \mathbb{R}
$$

From (3.9) and (3.17), we deduce that

$$
I\left(u^{t}\right) \geq \frac{a t^{2}}{2}\|\nabla u\|_{2}^{2}+\frac{b t^{4}}{4}\|\nabla u\|_{2}^{4}-t^{4} \int_{\mathbb{R}^{3}} K(x) F(u) \mathrm{d} x, \quad \forall t \in(0,1),
$$

which implies that $\zeta(t)>0$ for $t>0$ small. Moreover, by (K1), (K2), (F1), (F2) and (3.9), it is easy to verify that $\lim _{t \rightarrow 0} \zeta(t)=0$ and $\zeta(t)<0$ for $t$ large. Therefore $\max _{t \in(0, \infty)} \zeta(t)$ is achieved at $t_{u}>0$ so that $\zeta^{\prime}\left(t_{u}\right)=0$ and $u^{t_{u}} \in \mathcal{M}_{c}$.

Next, we claim that $t_{u}$ is unique for any $u \in H^{1}\left(\mathbb{R}^{3}\right) \backslash\{0\}$. Otherwise, for any given $u \in H^{1}\left(\mathbb{R}^{3}\right) \backslash\{0\}$, there exist positive constants $t_{1} \neq t_{2}$ such that $u^{t_{1}}, u^{t_{2}} \in \mathcal{M}_{c}$, that is, $J\left(u^{t_{1}}\right)=J\left(u^{t_{2}}\right)=0$. Then (3.13) implies

$$
\begin{aligned}
I\left(u^{t_{1}}\right) & >I\left(u^{t_{2}}\right)+\frac{t_{1}^{4}-t_{2}^{4}}{t_{1}^{4}} J\left(u^{t_{1}}\right)=I\left(u^{t_{2}}\right) \\
& >I\left(u^{t_{1}}\right)+\frac{t_{2}^{4}-t_{1}^{4}}{t_{2}^{4}} J\left(u^{t_{2}}\right)=I\left(u^{t_{1}}\right) .
\end{aligned}
$$

This contradiction shows that $t_{u}>0$ is unique for any $u \in H^{1}\left(\mathbb{R}^{3}\right) \backslash\{0\}$.

Combining Corollary 3.5 with Lemma 3.6, we obtain the following property. 
Lemma 3.7 Assume that hypotheses (K1), (K2) and (F1)-(F3) hold. Then

$$
\inf _{u \in \mathcal{M}_{c}} I(u)=m(c)=\inf _{u \in \mathcal{S}_{c}} \max _{t>0} I\left(u^{t}\right)
$$

Lemma 3.8 Assume that hypotheses (K1), (K2) and (F1)-(F3) hold. Then

(i) there exists $\rho_{0}>0$ such that $\|\nabla u\|_{2} \geq \rho_{0}, \forall u \in \mathcal{M}_{c}$;

(ii) $m(c)=\inf _{u \in \mathcal{M}_{c}} I(u)>0$.

Proof (i) By (F1), we deduce that

$$
\frac{F(t)}{|t|^{\mu-1} t} \text { is nonincreasing on both }(-\infty, 0) \text { and }(0,+\infty) \text {. }
$$

From (3.6) and (3.20), we derive that for any $s \in \mathbb{R}$,

$$
\begin{cases}|t|^{\mu} F(s) \leq F(s t) \leq|t|^{\frac{14}{3}} F(s), & \text { if }|t| \leq 1 \\ |t|^{\frac{14}{3}} F(s) \leq F(s t) \leq|t|^{\mu} F(s), & \text { if }|t| \geq 1\end{cases}
$$

which implies that there is a constant $C_{0}>0$ such that

$$
0 \leq F(t) \leq C_{0}\left(|t|^{\frac{14}{3}}+|t|^{\mu}\right), \quad \forall t \in \mathbb{R}
$$

By the Gagliardo-Nirenberg inequality, we have

$$
\|u\|_{s}^{s} \leq \mathcal{C}(s)\|\nabla u\|_{2}^{\frac{3(s-2)}{2}}\|u\|_{2}^{\frac{6-s}{2}}, \quad \forall s \in(2,6) .
$$

Since $J(u)=0, \forall u \in \mathcal{M}_{c}$, by (K1), (K2), (3.11), (3.22), (3.23) and the Sobolev inequality, we deduce that

$$
\begin{aligned}
a\|\nabla u\|_{2}^{2} & \leq a\|\nabla u\|_{2}^{2}+b\|\nabla u\|_{2}^{4} \\
& =2 \int_{\mathbb{R}^{3}} K(x)[f(u) u-2 F(u)] \mathrm{d} x-2 \int_{\mathbb{R}^{3}} \nabla K(x) \cdot x F(u) \mathrm{d} x \\
& \leq C_{1}\left(\|u\|_{\frac{14}{3}}^{\frac{14}{3}}+\|u\|_{6}^{6}\right) \\
& \leq C_{2}\|\nabla u\|_{2}^{4}\|u\|_{2}^{2 / 3}+C_{1} S^{-3}\|\nabla u\|_{2}^{6} \\
& =C_{2} c^{1 / 3}\|\nabla u\|_{2}^{4}+C_{1} S^{-3}\|\nabla u\|_{2}^{6}, \quad \forall u \in \mathcal{M}_{c},
\end{aligned}
$$

which concludes the proof of (i).

(ii) By (i) and (3.13) with $t \rightarrow 0$, we have

$$
I(u)=I(u)-\frac{1}{4} J(u) \geq \frac{a}{4}\|\nabla u\|_{2}^{2} \geq \frac{a}{4} \rho_{0}^{2}, \quad \forall u \in \mathcal{M}_{c} .
$$

Hence, $m(c)=\inf _{u \in \mathcal{M}_{c}} I(u)>0$. 
Lemma 3.9 Assume that hypotheses (K1), (K2) and (F1)-(F3) hold. Then $m(c)$ is nonincreasing on $(0, \infty)$. In particular, if $m(c)$ is achieved, then $m(c)>m(\tilde{c})$ for any $\tilde{c}>c$.

Proof For any $c_{2}>c_{1}>0$, there exists $\left\{u_{n}\right\} \subset \mathcal{M}_{c_{1}}$ such that

$$
I\left(u_{n}\right)<m\left(c_{1}\right)+\frac{1}{n}
$$

Let $\xi=\sqrt{c_{2} / c_{1}} \in(1, \infty)$ and $v_{n}(x)=\xi^{-1 / 2} u_{n}\left(\xi^{-1} x\right)$. Then $\left\|v_{n}\right\|_{2}^{2}=c_{2}$ and $\left\|\nabla v_{n}\right\|_{2}=\left\|\nabla u_{n}\right\|_{2}$. By Lemma 3.6, there exists $t_{n}>0$ such that $\left(v_{n}\right)^{t_{n}} \in \mathcal{M}_{c_{2}}$. Note that since $(6-\mu) K(x)+\nabla K(x) \cdot x \geq 0$ for all $x \in \mathbb{R}^{3}$ then

$$
t \mapsto t^{\frac{6-\mu}{2}} K(t x) \text { is nondecreasing on }(0,+\infty) \text { for every } x \in \mathbb{R}^{3} \text {. }
$$

Next, by (K2), (3.9), (3.13), (3.20) and (3.26), it follows that

$$
\begin{aligned}
& m\left(c_{2}\right) \\
& \leq I\left(\left(v_{n}\right)^{t_{n}}\right) \\
& =\frac{a t_{n}^{2}}{2}\left\|\nabla u_{n}\right\|_{2}^{2}+\frac{b t_{n}^{4}}{4}\left\|\nabla u_{n}\right\|_{2}^{4}-t_{n}^{-3} \xi^{3} \int_{\mathbb{R}^{3}} K\left(t_{n}^{-1} \xi x\right) F\left(t_{n}^{3 / 2} \xi^{-1 / 2} u_{n}\right) \mathrm{d} x \\
& \leq I\left(\left(u_{n}\right)^{t_{n}}\right)+t_{n}^{-3} \int_{\mathbb{R}^{3}}\left[K\left(t_{n}^{-1} x\right) F\left(t_{n}^{3 / 2} u_{n}\right)-\xi^{\frac{6-\mu}{2}} K\left(t_{n}^{-1} \xi x\right) \xi^{\frac{\mu}{2}} F\left(t_{n}^{3 / 2} \xi^{-1 / 2} u_{n}\right)\right] \mathrm{d} x \\
& \leq I\left(u_{n}\right)-\frac{a\left(1-t_{n}^{2}\right)^{2}}{4}\left\|\nabla u_{n}\right\|_{2}^{2}<m\left(c_{1}\right)+\frac{1}{n},
\end{aligned}
$$

which shows that $m\left(c_{2}\right) \leq m\left(c_{1}\right)$ by letting $n \rightarrow \infty$.

We now assume that $m(c)$ is achieved, that is, there exists $u \in \mathcal{M}_{c}$ such that $I(u)=m(c)$ for any given $c<\tilde{c}$. Let $\tilde{\xi}=\tilde{c} / c \in(1, \infty)$ and $v(x)=\tilde{\xi}^{-1 / 2} u\left(\tilde{\xi}^{-1} x\right)$. Then $\|v\|_{2}^{2}=\tilde{c}$ and $\|\nabla v\|_{2}=\|\nabla u\|_{2}$. By Lemma 3.6, there exists $\tilde{t}>0$ such that $v^{\tilde{t}} \in \mathcal{M}_{\tilde{c}}$. Then it follows from (K2), (3.9), (3.13), (3.20) and (3.26) that

$$
\begin{aligned}
m(\tilde{c}) & \leq I\left(v^{\tilde{t}}\right) \\
& =\frac{a \tilde{t}^{2}}{2}\|\nabla u\|_{2}^{2}+\frac{b \tilde{t}^{4}}{4}\|\nabla u\|_{2}^{4}-\tilde{t}^{-3} \tilde{\xi}^{3} \int_{\mathbb{R}^{3}} K\left(\tilde{t}^{-1} \tilde{\xi} x\right) F\left(\tilde{t}^{3 / 2} \tilde{\xi}^{-1 / 2} u\right) \mathrm{d} x \\
& \leq I\left(u^{\tilde{t}}\right)+\tilde{t}^{-3} \int_{\mathbb{R}^{3}}\left[K\left(\tilde{t}^{-1} x\right) F\left(\tilde{t}^{3 / 2} u\right)-\tilde{\xi}^{\frac{6-\mu}{2}} K\left(\tilde{t}^{-1} \tilde{\xi} x\right) \tilde{\xi}^{\frac{\mu}{2}} F\left(\tilde{t}^{3 / 2} \tilde{\xi}^{-1 / 2} u\right)\right] \mathrm{d} x \\
& \leq I(u)-\frac{a\left(1-\tilde{t}^{2}\right)^{2}}{4}\|\nabla u\|_{2}^{2}<m(c) .
\end{aligned}
$$

The proof is completed.

Remark 3.10 Lemmas 3.1-3.4 provide technical ingredients allowing generalization of the previous results to a new scenario when $K(x)$ is variable and $f(u)$ has a more 
general structure. But it is still difficult to prove that $m(c)$ is strictly decreasing on $(0, \infty)$ in the same way as the proof of [29, Lemma 4.5], because it is still unknown that $\left\{t_{n}\right\}$, involved in the proof of Lemma 3.9, is bounded from below for more general nonlinearity $f$.

Lemma 3.11 Assume that (K1), (K2) and (F1)-(F3) hold. Then $m(c) \leq m^{\infty}(c)$.

Proof In view of Lemmas 3.6 and 3.8, we have $\mathcal{M}_{c}^{\infty} \neq \emptyset$ and $m^{\infty}(c)>0$. Inspired by [8,25], assume by contradiction that $m(c)>m^{\infty}(c)$. Let $\varepsilon:=m(c)-m^{\infty}(c)$. Then there exists $u_{\varepsilon}^{\infty}$ such that

$$
u_{\varepsilon}^{\infty} \in \mathcal{M}_{c}^{\infty} \text { and } \quad m^{\infty}(c)+\frac{\varepsilon}{2}>I^{\infty}\left(u_{\varepsilon}^{\infty}\right)
$$

In view of Lemma 3.6, there exists $t_{\varepsilon}>0$ such that $\left(u_{\varepsilon}^{\infty}\right)^{t_{\varepsilon}} \in \mathcal{M}_{c}$. Since $K_{\infty} \leq K(x)$ for all $\mathbb{R}^{3}$, it follows from (1.3), (2.7), (3.27) and Corollary 3.5 that

$$
m^{\infty}(c)+\frac{\varepsilon}{2}>I^{\infty}\left(u_{\varepsilon}^{\infty}\right) \geq I^{\infty}\left(\left(u_{\varepsilon}^{\infty}\right)^{t_{\varepsilon}}\right) \geq I\left(\left(u_{\varepsilon}^{\infty}\right)^{t_{\varepsilon}}\right) \geq m(c) .
$$

This contradiction shows that $m(c) \leq m^{\infty}(c)$.

Similarly to [25, Lemma 2.7], [24, Lemma 2.10] and [27], we have the following Brezis-Lieb type lemma.

Lemma 3.12 Assume that hypotheses (K1), (K2), (F1) and (F2) hold. If $u_{n} \rightarrow \bar{u}$ in $H^{1}\left(\mathbb{R}^{3}\right)$, then

$$
I\left(u_{n}\right)=I(\bar{u})+I\left(u_{n}-\bar{u}\right)+\frac{b}{2}\|\nabla \bar{u}\|_{2}^{2}\left\|\nabla\left(u_{n}-\bar{u}\right)\right\|_{2}^{2}+o(1)
$$

and

$$
J\left(u_{n}\right)=J(\bar{u})+J\left(u_{n}-\bar{u}\right)+b\|\nabla \bar{u}\|_{2}^{2}\left\|\nabla\left(u_{n}-\bar{u}\right)\right\|_{2}^{2}+o(1) .
$$

Lemma 3.13 Assume that hypotheses (K1), (K2) and (F1)-(F3) hold. Then $m(c)$ is achieved.

Proof In view of Lemmas 3.6 and 3.8, we have $\mathcal{M}_{c} \neq \emptyset$ and $m(c)>0$. Let $\left\{u_{n}\right\} \subset \mathcal{M}_{c}$ be such that $I\left(u_{n}\right) \rightarrow m(c)$. Since $J\left(u_{n}\right)=0$, then it follows from (3.13) with $t \rightarrow 0$ that

$$
m(c)+o(1)=I\left(u_{n}\right) \geq \frac{a}{4}\left\|\nabla u_{n}\right\|_{2}^{2} .
$$

This relation together with $\left\|u_{n}\right\|_{2}^{2}=c$, implies that $\left\{u_{n}\right\}$ is bounded in $H^{1}\left(\mathbb{R}^{3}\right)$. Passing to a subsequence, we have $u_{n} \rightarrow \bar{u}$ in $H^{1}\left(\mathbb{R}^{3}\right)$. Then $u_{n} \rightarrow \bar{u}$ in $L_{\text {loc }}^{s}\left(\mathbb{R}^{3}\right)$ for $2 \leq s<6$ and $u_{n} \rightarrow \bar{u}$ a.e. in $\mathbb{R}^{3}$. There are two possible cases: i) $\bar{u}=0$ and ii) $\bar{u} \neq 0$.

Case i) $\bar{u}=0$, namely $u_{n} \rightarrow 0$ in $H^{1}\left(\mathbb{R}^{3}\right)$. Then $u_{n} \rightarrow 0$ in $L_{\text {loc }}^{s}\left(\mathbb{R}^{3}\right)$ for $2 \leq s<6$ and $u_{n} \rightarrow 0$ a.e. in $\mathbb{R}^{3}$. By (K1) and (3.3), it is easy to show that

$$
\lim _{n \rightarrow \infty} \int_{\mathbb{R}^{3}}\left[K_{\infty}-K(x)\right] F\left(u_{n}\right) \mathrm{d} x=\lim _{n \rightarrow \infty} \int_{\mathbb{R}^{3}} \nabla K(x) \cdot x F\left(u_{n}\right) \mathrm{d} x=0 .
$$


From (1.3), (2.7), (3.11), (3.12) and (3.31), we deduce that

$$
I^{\infty}\left(u_{n}\right) \rightarrow m(c), \quad J^{\infty}\left(u_{n}\right) \rightarrow 0 .
$$

From (3.12), (3.32), Lemmas 3.6 and 3.7 (i), we have

$$
a \rho_{0}^{2} \leq a\left\|\nabla u_{n}\right\|_{2}^{2}+b\left\|\nabla u_{n}\right\|_{2}^{4}=\frac{3}{2} \int_{\mathbb{R}^{3}} K_{\infty}\left[f\left(u_{n}\right) u_{n}-2 F\left(u_{n}\right)\right] \mathrm{dx} .
$$

Using (3.22), (3.33) and Lions' concentration-compactness principle [27, Lemma 1.21], we prove that there exist $\delta>0$ and $\left\{y_{n}\right\} \subset \mathbb{R}^{3}$ such that $\int_{B_{1}\left(y_{n}\right)}\left|u_{n}\right|^{2} \mathrm{~d} x>\delta$. Let $\hat{u}_{n}(x)=u_{n}\left(x+y_{n}\right)$. Then we have $\left\|\hat{u}_{n}\right\|=\left\|u_{n}\right\|$ and

$$
J^{\infty}\left(\hat{u}_{n}\right)=o(1), \quad I^{\infty}\left(\hat{u}_{n}\right) \rightarrow m(c), \quad \int_{B_{1}(0)}\left|\hat{u}_{n}\right|^{2} \mathrm{~d} x>\delta .
$$

Therefore, there exists $\hat{u} \in H^{1}\left(\mathbb{R}^{3}\right) \backslash\{0\}$ such that, up to a subsequence,

$$
\left\{\begin{array}{l}
\hat{u}_{n} \rightarrow \hat{u}, \quad \text { in } H^{1}\left(\mathbb{R}^{3}\right) ; \\
\hat{u}_{n} \rightarrow \hat{u}, \text { in } L_{\text {loc }}^{s}\left(\mathbb{R}^{3}\right), \forall s \in[1,6) ; \\
\hat{u}_{n} \rightarrow \hat{u}, \text { a.e. on } \mathbb{R}^{3} .
\end{array}\right.
$$

Let $w_{n}=\hat{u}_{n}-\hat{u}$. Then (3.35) and Lemma 3.12 yield

$$
\begin{aligned}
& \|\hat{u}\|_{2}^{2}:=\hat{c} \leq c, \quad\left\|w_{n}\right\|_{2}^{2}:=\hat{c}_{n} \leq c \text { for large } n \in \mathbb{N}, \\
& I^{\infty}\left(\hat{u}_{n}\right)=I^{\infty}(\hat{u})+I^{\infty}\left(w_{n}\right)+\frac{b}{2}\|\nabla \hat{u}\|_{2}^{2}\left\|\nabla w_{n}\right\|_{2}^{2}+o(1)
\end{aligned}
$$

and

$$
J^{\infty}\left(\hat{u}_{n}\right)=J^{\infty}(\hat{u})+J^{\infty}\left(w_{n}\right)+b\|\nabla \hat{u}\|_{2}^{2}\left\|\nabla w_{n}\right\|_{2}^{2}+o(1) .
$$

Let

$$
\begin{aligned}
\Psi^{\infty}(u) & :=I^{\infty}(u)-\frac{1}{4} J^{\infty}(u) \\
& =\frac{a}{4}\|\nabla u\|_{2}^{2}+\frac{1}{8} \int_{\mathbb{R}^{3}} K_{\infty}[3 f(u) u-14 F(u)] \mathrm{d} x, \quad \forall u \in H^{1}\left(\mathbb{R}^{3}\right)(3.39)
\end{aligned}
$$

By (3.7), we have $\Psi^{\infty}(u)>0$ for all $u \in H^{1}\left(\mathbb{R}^{3}\right) \backslash\{0\}$. Moreover, it follows from (3.34), (3.37), (3.38) and (3.39) that

$$
\Psi^{\infty}\left(w_{n}\right) \leq m(c)-\Psi^{\infty}(\hat{u})+o(1), \quad J^{\infty}\left(w_{n}\right) \leq-J^{\infty}(\hat{u})+o(1) .
$$

If there exists a subsequence $\left\{w_{n_{i}}\right\}$ of $\left\{w_{n}\right\}$ such that $w_{n_{i}}=0$, then it follows from (3.36), Lemmas 3.9 and 3.11 that

$$
m^{\infty}(\hat{c}) \leq I^{\infty}(\hat{u})=m(c) \leq m(\hat{c}) \leq m^{\infty}(\hat{c}), \quad J^{\infty}(\hat{u})=0,
$$


which, together with $m^{\infty}(c) \leq m^{\infty}(\hat{c}) \leq I^{\infty}(\hat{u})=m(c) \leq m^{\infty}(c)$, implies

$$
I^{\infty}(\hat{u})=m^{\infty}(\hat{c})=m(\hat{c})=m(c)=m^{\infty}(c), \quad J^{\infty}(\hat{u})=0 .
$$

Next, we assume that $w_{n} \neq 0$. We claim that $J^{\infty}(\hat{u}) \leq 0$. Otherwise, if $J^{\infty}(\hat{u})>0$, then (3.40) implies $J^{\infty}\left(w_{n}\right)<0$ for large $n$. In view of Lemma 3.6, there exists $t_{n}>0$ such that $\left(w_{n}\right)^{t_{n}} \in \mathcal{M}_{\hat{c}_{n}}^{\infty}$. Then it follows from (2.7), (3.12), (3.13), (3.39), (3.40), Lemmas 3.9 and 3.11 that

$$
\begin{aligned}
m(c)-\Psi^{\infty}(\hat{u})+o(1) & \geq \Psi^{\infty}\left(w_{n}\right)=I^{\infty}\left(w_{n}\right)-\frac{1}{4} J^{\infty}\left(w_{n}\right) \\
& \geq I^{\infty}\left(\left(w_{n}\right)^{t_{n}}\right)-\frac{t_{n}^{4}}{4} J^{\infty}\left(w_{n}\right) \\
& \geq m^{\infty}\left(\hat{c}_{n}\right)-\frac{t_{n}^{4}}{4} J^{\infty}\left(w_{n}\right) \\
& \geq m^{\infty}(c)+o(1) \geq m(c)+o(1),
\end{aligned}
$$

which is impossible due to $\Psi^{\infty}(\hat{u})>0$. This shows that $J^{\infty}(\hat{u}) \leq 0$. In view of Lemma 3.6, there exists $t_{\infty}>0$ such that $\hat{u}^{t_{\infty}} \in \mathcal{M}_{\hat{c}}^{\infty}$. From (2.7), (3.12), (3.13), (3.34), (3.39), the weak semicontinuity of the norm, Fatou's lemma, Lemmas 3.9 and 3.11 , one has

$$
\begin{aligned}
m(c) & =\lim _{n \rightarrow \infty}\left[I^{\infty}\left(\hat{u}_{n}\right)-\frac{1}{4} J^{\infty}\left(\hat{u}_{n}\right)\right] \\
& =\lim _{n \rightarrow \infty} \Psi^{\infty}\left(\hat{u}_{n}\right) \geq \Psi^{\infty}(\hat{u}) \\
& =I^{\infty}(\hat{u})-\frac{1}{4} J^{\infty}(\hat{u}) \geq I^{\infty}\left(\hat{u}^{t_{\infty}}\right)-\frac{t_{\infty}^{4}}{4} J^{\infty}(\hat{u}) \\
& \geq m^{\infty}(\hat{c})-\frac{t_{\infty}^{4}}{4} J^{\infty}(\hat{u}) \geq m(\hat{c}) \geq m(c),
\end{aligned}
$$

which implies that (3.41) holds for $w_{n} \neq 0$. From (3.36), (3.41), Lemmas 3.9 and 3.11, we deduce that

$$
m^{\infty}(c) \leq m^{\infty}(\hat{c})=I^{\infty}(\hat{u})=m(\hat{c})=m(c) \leq m^{\infty}(c), \quad J^{\infty}(\hat{u})=0,
$$

which implies that (3.41) holds for $w_{n} \neq 0$. Thus, $m^{\infty}(\hat{c})$ is achieved at $\hat{u}$. In view of Lemma 3.9, we deduce that $\|\hat{u}\|_{2}^{2}=\hat{c}=c$ due to $m^{\infty}(\hat{c})=m^{\infty}(c)$. By Lemma 3.6, there exists $\hat{t}>0$ such that $\hat{u}^{\hat{t}} \in \mathcal{M}_{c}$. Then it follows from (3.9), (3.13), (3.20), (3.26) and (3.41) that

$$
\begin{aligned}
m(c) & \leq I\left(\hat{u}^{\hat{t}}\right) \leq I^{\infty}\left(\hat{u}^{\hat{t}}\right) \\
& \leq I^{\infty}(\hat{u})-\frac{a\left(1-\hat{t}^{2}\right)^{2}}{4}\|\nabla \hat{u}\|_{2}^{2}=m(c)-\frac{a\left(1-\hat{t}^{2}\right)^{2}}{4}\|\nabla \hat{u}\|_{2}^{2},
\end{aligned}
$$


which implies that $\hat{u} \in \mathcal{M}_{c}$ and $I(\hat{u})=m(c)$. Hence, $m(c)$ is achieved at $\hat{u} \in \mathcal{M}_{c}$.

Case ii) $\bar{u} \neq 0$. Let $v_{n}=u_{n}-\bar{u}$. Then Lemma 3.12 yields

$$
\begin{aligned}
& \|\bar{u}\|_{2}^{2}:=\bar{c} \leq c, \quad\left\|v_{n}\right\|_{2}^{2}:=c_{n} \leq c \text { for large } n \in \mathbb{N}, \\
& I\left(u_{n}\right)=I(\bar{u})+I\left(v_{n}\right)+\frac{b}{2}\|\nabla \bar{u}\|_{2}^{2}\left\|\nabla v_{n}\right\|_{2}^{2}+o(1)
\end{aligned}
$$

and

$$
J\left(u_{n}\right)=J(\bar{u})+J\left(v_{n}\right)+b\|\nabla \bar{u}\|_{2}^{2}\left\|\nabla v_{n}\right\|_{2}^{2}+o(1) .
$$

Let

$$
\begin{aligned}
\Psi(u):= & I(u)-\frac{1}{4} J(u) \\
= & \frac{a}{4}\|\nabla u\|_{2}^{2}+\frac{1}{8} \int_{\mathbb{R}^{3}} K(x)[3 f(u) u-14 F(u)] \mathrm{d} x \\
& \quad-\frac{1}{4} \int_{\mathbb{R}^{3}} \nabla K(x) \cdot x F(u) \mathrm{d} x, \quad \forall u \in H^{1}\left(\mathbb{R}^{3}\right) .
\end{aligned}
$$

By (3.4) and (3.7), one has $\Psi(u)>0$ for all $u \in H^{1}\left(\mathbb{R}^{3}\right) \backslash\{0\}$. Similarly to the proof of (3.40), we deduce that

$$
\Psi\left(v_{n}\right) \leq m(c)-\Psi(\bar{u})+o(1), \quad J\left(v_{n}\right) \leq-J(\bar{u})+o(1) .
$$

If there exists a subsequence $\left\{v_{n_{i}}\right\}$ of $\left\{v_{n}\right\}$ such that $v_{n_{i}}=0$, then it follows from Lemma 3.9 that

$$
m(\bar{c}) \leq I(\bar{u})=m(c) \leq m(\bar{c}), \quad J(\bar{u})=0,
$$

which implies

$$
I(\bar{u})=m(c)=m(\bar{c}), \quad J(\bar{u})=0 .
$$

Next, we prove that (3.47) holds for $v_{n} \neq 0$. To this end, we assume that $v_{n} \neq 0$. We claim that $J(\bar{u}) \leq 0$. Otherwise $J(\bar{u})>0$, then (3.46) implies $J\left(v_{n}\right)<0$ for large $n$. In view of Lemma 3.6, there exists $t_{n}>0$ such that $\left(v_{n}\right)^{t_{n}} \in \mathcal{M}_{c_{n}}$. From (3.13), (3.45) and (3.46), we obtain

$$
\begin{aligned}
m(c)-\Psi(\bar{u})+o(1) & \geq \Psi\left(v_{n}\right)=I\left(v_{n}\right)-\frac{1}{4} J\left(v_{n}\right) \\
& \geq I\left(\left(v_{n}\right)^{t_{n}}\right)-\frac{t_{n}^{4}}{4} J\left(v_{n}\right) \geq m\left(\bar{c}_{n}\right) \geq m(c)+o(1),
\end{aligned}
$$

which is impossible due to $\Psi(\bar{u})>0$. This shows that $J(\bar{u}) \leq 0$. In view of Lemma 3.6, there exists $\tilde{t}>0$ such that $\bar{u}^{\tilde{t}} \in \mathcal{M}_{\bar{c}}$. Then it follows from (3.13), (3.45), the weak semicontinuity of norm, Fatou's lemma and Lemma 3.11 that 


$$
\begin{aligned}
m(c) & =\lim _{n \rightarrow \infty}\left[I\left(u_{n}\right)-\frac{1}{4} J\left(u_{n}\right)\right]=\lim _{n \rightarrow \infty} \Psi\left(u_{n}\right) \\
& \geq \Psi(\bar{u})=I(\bar{u})-\frac{1}{4} J(\bar{u}) \\
& \geq I\left(\bar{u}^{\tilde{t}}\right)-\frac{\tilde{t}^{4}}{4} J(\bar{u}) \geq m(\bar{c}) \geq m(c),
\end{aligned}
$$

which implies (3.47) holds for $v_{n} \neq 0$. This shows that $m(\bar{c})$ is achieved at $\bar{u} \in \mathcal{M}_{\bar{c}}$. In view of Lemma 3.9, we have $\|\bar{u}\|_{2}^{2}=\bar{c}=c$ due to $m(c)=m(\bar{c})$. By Lemma 3.6, there exists $\bar{t}>0$ such that $\bar{u}^{\bar{t}} \in \mathcal{M}_{c}$. Then it follows from (3.9), (3.13), (3.20), (3.26) and (3.47) that

$$
\begin{aligned}
m(c) & \leq I\left(\bar{u}^{\bar{t}}\right) \\
& \leq I(\bar{u})-\frac{a\left(1-\bar{t}^{2}\right)^{2}}{4}\|\nabla \bar{u}\|_{2}^{2}=m(c)-\frac{a\left(1-\bar{t}^{2}\right)^{2}}{4}\|\nabla \bar{u}\|_{2}^{2},
\end{aligned}
$$

which implies that $\bar{u} \in \mathcal{M}_{c}$ and $I(\bar{u})=m(c)$. Hence, $m(c)$ is achieved at $\bar{u} \in \mathcal{M}_{c}$.

Inspired by [8, Lemma 2.14], we prove the following lemma.

Lemma 3.14 Assume that hypotheses (K1), (K2) and (F1)-(F3) hold. If $\bar{u} \in \mathcal{M}_{c}$ and $I(\bar{u})=m(c)$, then $\bar{u}$ is a critical point of $\left.I\right|_{\mathcal{S}_{c}}$.

Proof Assume that $\left.I\right|_{\mathcal{S}_{c}} ^{\prime}(\bar{u}) \neq 0$. Then there exist $\delta>0$ and $\varrho>0$ such that

$$
u \in \mathcal{S}_{c}, \quad\|u-\bar{u}\| \leq 3 \delta \Rightarrow\left\|\left.I\right|_{\mathcal{S}_{c}} ^{\prime}(u)\right\| \geq \varrho
$$

Similarly to $[25,(2.59)]$, we prove that

$$
\lim _{t \rightarrow 1}\left\|\bar{u}^{t}-\bar{u}\right\|=0
$$

Thus, there exists $\delta_{1} \in(0,1 / 4)$ such that

$$
|t-1|<\delta_{1} \Rightarrow\left\|\bar{u}^{t}-\bar{u}\right\|<\delta
$$

In view of (3.13), we have

$$
I\left(\bar{u}^{t}\right) \leq I(\bar{u})-\frac{a\left(1-t^{2}\right)^{2}}{4}\|\nabla \bar{u}\|_{2}^{2}=m(c)-\frac{a\left(1-t^{2}\right)^{2}}{4}\|\nabla \bar{u}\|_{2}^{2}, \quad \forall t>0 .
$$

From (F3), (3.2), (3.11), (3.16) and (3.17), we deduce that there exist $T_{1} \in(0,1)$ and $T_{2} \in(1, \infty)$ such that

$$
J\left(\bar{u}^{T_{1}}\right)>0, \quad J\left(\bar{u}^{T_{2}}\right)<0
$$


Let $\varepsilon:=\min \left\{a\left(1-T_{1}^{2}\right)^{2}\|\nabla \bar{u}\|_{2}^{2} / 12, a\left(1-T_{2}^{2}\right)^{2}\|\nabla \bar{u}\|_{2}^{2} / 12,1, \varrho \delta / 8\right\}$ and $\mathcal{S}:=$ $B(\bar{u}, \delta) \cap \mathcal{S}_{c}$. By [27, Lemma 2.3] or [23], there exists a deformation $\eta \in \mathcal{C}([0,1] \times$ $\left.\mathcal{S}_{c}, \mathcal{S}_{c}\right)$ such that

(i) $\eta(1, u)=u$ if $I(u)<m(c)-2 \varepsilon$ or $I(u)>m(c)+2 \varepsilon$;

(ii) $\eta\left(1, I^{m(c)+\varepsilon} \cap \mathcal{S}\right) \subset I^{m(c)-\varepsilon}$;

(iii) $I(\eta(1, u)) \leq I(u), \forall u \in \mathcal{S}_{c}$;

(iv) $\eta(1, u)$ is a homeomorphism of $\mathcal{S}_{c}$.

By Corollary 3.5, I $\left(\bar{u}^{t}\right) \leq I(\bar{u})=m(c)$ for $t>0$. Thus, by (3.50) and ii) we obtain

$$
I\left(\eta\left(1, \bar{u}^{t}\right)\right) \leq m(c)-\varepsilon, \quad \forall t>0, \quad|t-1|<\delta_{1} .
$$

On the other hand, by iii) and (3.51), one has

$$
\begin{aligned}
I\left(\eta\left(1, \bar{u}^{t}\right)\right) & \leq I\left(\bar{u}^{t}\right) \\
& \leq m(c)-\frac{a\left(1-t^{2}\right)^{2}}{4}\|\nabla \bar{u}\|_{2}^{2} \\
& \leq m(c)-\delta_{2}\|\nabla \bar{u}\|_{2}^{2}, \quad \forall t>0, \quad|t-1| \geq \delta_{1},
\end{aligned}
$$

where

$$
\delta_{2}:=\frac{a}{4} \min \left\{\left(1-T_{1}^{2}\right)^{2},\left(1-T_{1}^{2}\right)^{2}\right\}>0 .
$$

Combining (3.53) with (3.54), we have

$$
\max _{t \in\left[T_{1}, T_{2}\right]} I\left(\eta\left(1, \bar{u}^{t}\right)\right)<m(c) .
$$

Define $\Psi_{0}(t):=J\left(\eta\left(1, \bar{u}^{t}\right)\right)$ for $t>0$. It follows from (3.51) and i) that $\eta\left(1, \bar{u}^{t}\right)=$ $\bar{u}^{t}$ for $t=T_{1}$ and $t=T_{2}$, which, together with (3.52), implies

$$
\Psi_{0}\left(T_{1}\right)=J\left(\bar{u}^{T_{1}}\right)>0, \quad \Psi_{0}\left(T_{2}\right)=J\left(\bar{u}^{T_{1}}\right)<0 .
$$

Since $\Psi_{0}(t)$ is continuous on $(0, \infty)$, then $\eta\left(1, \bar{u}^{t}\right) \cap \mathcal{M}_{c} \neq \emptyset$ for some $t_{0} \in\left[T_{1}, T_{2}\right]$, contradicting the definition of $m(c)$.

Lemma 3.15 Assume that hypotheses (K1), (K2) and (F1)-(F3) hold. If $\bar{u} \in \mathcal{S}_{c}$ is a critical point of $\left.I\right|_{\mathcal{S}_{c}}$, then $J(\bar{u})=0$, and there exists $\lambda_{c}<0$ such that $I^{\prime}(\bar{u})-\lambda_{c} u=0$. 
Proof Since $\left(\left.I\right|_{\mathcal{S}_{c}}\right)^{\prime}(\bar{u})=0$, there exists $\lambda_{c} \in \mathbb{R}$ such that $I^{\prime}(\bar{u})-\lambda_{c} \bar{u}=0$, and so

$$
\left\langle I^{\prime}(\bar{u})-\lambda_{c} \bar{u}, u\right\rangle=a\|\nabla \bar{u}\|_{2}^{2}+b\|\nabla \bar{u}\|_{2}^{4}-\int_{\mathbb{R}^{3}} K(x) f(\bar{u}) \bar{u} \mathrm{~d} x-\lambda_{c}\|\bar{u}\|_{2}^{2}=0 .
$$

Moreover, $\bar{u}$ satisfies the following Pohozaev identity:

$$
\mathcal{P}(\bar{u}):=\frac{a}{2}\|\nabla \bar{u}\|_{2}^{2}+\frac{b}{2}\|\nabla \bar{u}\|_{2}^{4}-\int_{\mathbb{R}^{3}}[3 K(x)+\nabla K(x) \cdot x] F(\bar{u}) \mathrm{d} x-\frac{3 \lambda_{c}}{2}\|\bar{u}\|_{2}^{2}=0 .
$$

Then relations (3.11), (3.56) and (3.57) yield

$$
J(\bar{u})=\frac{3}{2}\left\langle I^{\prime}(\bar{u})-\lambda_{c} \bar{u}, \bar{u}\right\rangle-\mathcal{P}(\bar{u})=0 .
$$

Noting that $\|\bar{u}\|_{2}^{2}=c$, it follows from (K2), (F1), (3.56) and (3.57) that

$$
\begin{aligned}
2 \lambda c & =\int_{\mathbb{R}^{3}}\{K(x) f(\bar{u}) \bar{u}-[6 K(x)+2 \nabla K(x) \cdot x] F(\bar{u})\} \mathrm{d} x \\
& =\int_{\mathbb{R}^{3}}\{K(x)[f(\bar{u}) \bar{u}-\mu F(\bar{u})]-[(6-\mu) K(x)+2 \nabla K(x) \cdot x] F(\bar{u})\} \mathrm{d} x<0,
\end{aligned}
$$

and so $\lambda_{c}<0$. This completes the proof.

Proof of Theorem 2.1 In view of Lemmas 3.7, 3.8 and 3.13-3.15, for any $c>0$ there exists $\bar{u}_{c} \in \mathcal{M}_{c}$ such that

$$
I\left(\bar{u}_{c}\right)=m(c)=\inf _{u \in \mathcal{S}_{c}} \max _{t>0} I\left(\left(\bar{u}_{c}\right)^{t}\right)>0, \quad I^{\prime}\left(\bar{u}_{c}\right)=0
$$

and there exists Lagrange multiplier $\lambda_{c} \in \mathbb{R}^{-}$such that $\left(\bar{u}_{c}, \lambda_{c}\right)$ is a solution of problem (1.1).

\section{Second Existence Result and Qualitative Properties of Solutions}

In this section, we give the proof of Theorem 2.2.

\subsection{Global Minimizers on the Constraint $\mathcal{S}_{c}$}

Lemma 4.1 Assume that hypotheses (K1) and (F4) hold. Then

(i) for any $c>0, \sigma(c)=\inf _{u \in \mathcal{S}_{c}} I(u)$ is well defined and $\sigma(c) \leq 0$;

(ii) for any $c>0, \sigma(c)<0$ if (F6) holds;

(iii) there exists $\mathcal{C}_{0}>0$ such that $\sigma(c)<0$ for any $c>\mathcal{C}_{0}$ if (F5) holds. 
Proof (i) Using (F4), for any $\varepsilon>0$, there exists $C_{\varepsilon}>0$ such that

$$
|f(t) t|+|F(t)| \leq \varepsilon|t|^{2}+C_{\varepsilon}|t|^{p}, \quad \forall t \in \mathbb{R}
$$

By (1.3), (3.23) and (4.1), we have

$$
\begin{aligned}
I(u) \geq & \frac{a}{2}\|\nabla u\|_{2}^{2}+\frac{b}{4}\|\nabla u\|_{2}^{4}-\sup _{\mathbb{R}^{3}} K\left(\varepsilon\|u\|_{2}^{2}+C_{\varepsilon}\|u\|_{p}^{p}\right) \\
\geq & \frac{a}{2}\|\nabla u\|_{2}^{2}+\frac{b}{4}\|\nabla u\|_{2}^{4}-\sup _{\mathbb{R}^{3}} K\left(\varepsilon\|u\|_{2}^{2}+C_{\varepsilon} \mathcal{C}(p)\|\nabla u\|_{2}^{\frac{3(p-2)}{2}}\|u\|_{2}^{\frac{6-p}{2}}\right), \\
& \forall u \in \mathcal{S}_{c}, c>0 .
\end{aligned}
$$

This relation together with $0<3(p-2) / 2<4$, shows that $I$ is bounded from below on $\mathcal{S}_{c}$ for any $c>0$, that is, $\sigma(c)$ is well defined. Noting that $u^{t} \in \mathcal{S}_{c}$ for all $u \in \mathcal{S}_{c}$, from (3.9) and (4.1), we deduce that $I\left(u^{t}\right) \rightarrow 0$ as $t \rightarrow 0$, and so $\sigma(c) \leq 0$ for any $c>0$.

(ii) By (F4) and (F6), there exist $\delta_{0}, \varrho_{0}>0$ such that

$$
|F(t)| \geq \delta_{0}|t|^{q_{0}}, \quad \forall|t| \leq \varrho_{0} .
$$

For any $c>0$, we choose a function $u_{0} \in \mathcal{C}_{0}^{\infty}\left(\mathbb{R}^{3},\left[-\varrho_{0}, \varrho_{0}\right]\right)$ satisfying $\left\|u_{0}\right\|_{2}^{2}=c$. Then it follows from (3.9) and (4.3) that

$$
I\left(u_{0}^{t}\right) \leq \frac{a t^{2}}{2}\left\|\nabla u_{0}\right\|_{2}^{2}+\frac{b t^{4}}{4}\left\|\nabla u_{0}\right\|_{2}^{4}-K_{\infty} \delta_{0} t^{3\left(q_{0}-2\right) / 2}\left\|u_{0}\right\|_{q_{0}}^{q_{0}}, \quad \forall 0<t \leq 1
$$

Since $0<3\left(q_{0}-2\right) / 2<2$, (4.4) implies that $I\left(u_{0}{ }^{t}\right)<0$ for small $t \in(0,1)$. Jointly with the fact that $\left\|u_{0}{ }^{t}\right\|_{2}=\left\|u_{0}{ }^{t}\right\|_{2}$, we have $\sigma(c) \leq \inf _{t \in(0,1]} I\left(u_{0}{ }^{t}\right)<0$.

(iii) We set the following scaling

$$
u_{t}(x):=t^{1 / 2} u(x / t), \quad \forall u \in H^{1}\left(\mathbb{R}^{3}\right), t>0 .
$$

Note that (F5) implies

$$
\frac{F(t)}{|t|^{\mu_{0}-1} t} \text { is nondecreasing on }(-\infty, 0) \text { and }(0, \infty) \text {. }
$$

For any $u \in \mathcal{S}_{1}$, by (1.3), (4.5) and (4.6), we have

$$
\begin{aligned}
I\left(u_{t}\right) & =\frac{a t^{2}}{2}\|\nabla u\|_{2}^{2}+\frac{b t^{4}}{4}\|\nabla u\|_{2}^{4}-t^{3} \int_{\mathbb{R}^{3}} K(t x) F\left(t^{1 / 2} u\right) \mathrm{d} x \\
& \leq \frac{a t^{2}}{2}\|\nabla u\|_{2}^{2}+\frac{b t^{4}}{4}\|\nabla u\|_{2}^{4}-K_{\infty} t^{3+\mu_{0} / 2} \int_{\mathbb{R}^{3}} F(u) \mathrm{d} x, \quad \forall t>1,
\end{aligned}
$$


which, together with $3+\mu_{0} / 2>4$, implies that $I\left(u_{t}\right) \rightarrow-\infty$ as $t \rightarrow+\infty$. Since $\left\|u_{t}\right\|_{2}^{2}=t^{4}\|u\|_{2}^{2}=t^{4}$ for $u \in \mathcal{S}_{1}$ and $t>0$, there exists $\mathcal{C}_{0}>0$ such that $\sigma(c)<0$ for any $c>\mathcal{C}_{0}$.

Noting that Lemma 4.1 implies

$$
\{c \in(0,+\infty), \sigma(c)<0\} \neq \emptyset,
$$

we have

$$
c_{*}=\inf \{c \in(0,+\infty), \sigma(c)<0\}
$$

is well-defined.

Lemma 4.2 Assume that hypotheses (K1) and (F4) hold. Then the following properties hold:

(i) if (F6) holds, then $c_{*}=0$;

(ii) if (F5) and $\left(F 6^{\prime}\right)$ hold, then $c_{*} \in(0,+\infty)$; moreover, $\sigma(c)=0$ for any $c \in\left(0, c_{*}\right]$ and $\sigma(c)<0$ for any $c>c_{*}$.

Proof (i) Obviously, (i) follows directly from Lemma 4.1 (ii).

(ii) We first prove that $c^{*}>0$. From (F4), (F6') and (3.23), we obtain

$$
\begin{aligned}
\int_{\mathbb{R}^{3}} F(u) \mathrm{d} x & \leq C_{3}\left(\|u\|_{\frac{10}{3}}^{\frac{10}{3}}+\|u\|_{p}^{p}\right) \\
& \leq C_{4}\left(\|\nabla u\|_{2}^{2}\|u\|_{2}^{\frac{4}{3}}+\|\nabla u\|_{2}^{\frac{3(p-2)}{2}}\|u\|_{2}^{\frac{6-p}{2}}\right), \quad \forall u \in H^{1}\left(\mathbb{R}^{3}\right) .
\end{aligned}
$$

It is easy to check that there exists a constant $\epsilon>0$ small enough such that

$$
\epsilon^{\frac{2}{3}} \leq \frac{a}{4 C_{4}} \text { and } \epsilon^{\frac{6-p}{14-3 p}} \leq C_{4}^{\frac{4}{3 p-14}} \frac{4 a}{14-3 p}\left(\frac{4 b}{3 p-10}\right)^{\frac{3 p-10}{14-3 p}}
$$

For any $u \in \mathcal{S}_{\epsilon}$, namely $\|u\|_{2}^{2}=\epsilon$, it follows from (1.3), (4.9) and (4.10) that

$$
\begin{aligned}
I(u) & \geq \frac{a}{2}\|\nabla u\|_{2}^{2}+\frac{b}{4}\|\nabla u\|_{2}^{4}-C_{4}\left(\|u\|_{2}^{\frac{4}{3}}\|\nabla u\|_{2}^{2}+\|u\|_{2}^{\frac{6-p}{2}}\|\nabla u\|_{2}^{\frac{3(p-2)}{2}}\right) \\
& \geq \frac{1}{4}\|\nabla u\|_{2}^{2}\left(a+b\|\nabla u\|_{2}^{2}-C_{4} \epsilon^{\frac{6-p}{4}}\|\nabla u\|_{2}^{\frac{3 p-10}{2}}\right),
\end{aligned}
$$


By Young's inequality and (4.10), we have

$$
\begin{aligned}
C_{4} \epsilon^{\frac{6-p}{4}}\|\nabla u\|_{2}^{\frac{3 p-10}{2}} & =\left(\frac{4 b}{3 p-10}\right)^{\frac{3 p-10}{4}}\|\nabla u\|_{2}^{\frac{3 p-10}{2}} \cdot\left(\frac{3 p-10}{4 b}\right)^{\frac{3 p-10}{4}} C_{4} \epsilon^{\frac{6-p}{4}} \\
& \leq b\|\nabla u\|_{2}^{2}+C_{4}^{\frac{4}{14-3 p}} \frac{14-3 p}{4}\left(\frac{3 p-10}{4 b}\right)^{\frac{3 p-10}{14-3 p}} \epsilon^{\frac{6-p}{14-3 p}} \\
& \leq b\|\nabla u\|_{2}^{2}+a
\end{aligned}
$$

which, together with (4.11), leads to $I(u) \geq 0$ for $u \in \mathcal{S}_{\epsilon}$. On the other hand, by Lemma 4.1 (i), one has $\sigma(c) \leq 0$ for $c>0$. This shows that $c_{*}=$ $\inf \{c \in(0,+\infty), \sigma(c)<0\}>0$. Moreover, from the definition of $c_{*}$ and Lemma 4.1 (i), we deduce that $\sigma(c)=0$ for any $c \in\left(0, c_{*}\right]$ and $\sigma(c)<0$ for any $c>c_{*}$.

Lemma 4.3 Assume that (K1), (K3), (F4), (F5) and either of (F6) and (F6') hold. Then

(i) for any $c>0, \sigma(c)$ is continuous;

(ii) for any $c>c_{*}$,

$$
\sigma(c)<\sigma(\alpha)+\sigma(c-\alpha), \quad \forall 0<\alpha<c .
$$

Proof (i) For any $c>0$, let $c_{n}>0$ and $c_{n} \rightarrow c$. For every $n \in \mathbb{N}$, let $u_{n} \in \mathcal{S}_{c_{n}}$ such that $I\left(u_{n}\right)<\sigma\left(c_{n}\right)+\frac{1}{n} \leq \frac{1}{n}$. Then (4.2) implies that $\left\{u_{n}\right\}$ is bounded in $H^{1}\left(\mathbb{R}^{3}\right)$, moreover, we have

$$
\sigma(c) \leq I\left(\sqrt{\frac{c}{c_{n}}} u_{n}\right)=I\left(u_{n}\right)+o(1) \leq \sigma\left(c_{n}\right)+o(1) .
$$

On the other hand, given a minimization sequence $\left\{v_{n}\right\} \subset \mathcal{S}_{c}$ for $I$, we have

$$
\sigma\left(c_{n}\right) \leq I\left(\sqrt{\frac{c_{n}}{c}} v_{n}\right) \leq I\left(v_{n}\right)+o(1)=\sigma(c)+o(1),
$$

which, jointly to (4.14), gives $\lim _{n \rightarrow \infty} \sigma\left(c_{n}\right)=\sigma(c)$.

(ii) Note that (1.3) and (4.6) lead to

$$
\begin{aligned}
I\left(u_{t}\right) & =\frac{a t^{2}}{2}\|\nabla u\|_{2}^{2}+\frac{b t^{4}}{4}\|\nabla u\|_{2}^{4}-t^{3} \int_{\mathbb{R}^{3}} K(t x) F\left(t^{1 / 2} u\right) \mathrm{d} x \\
& \leq \frac{a t^{2}}{2}\|\nabla u\|_{2}^{2}+\frac{b t^{4}}{4}\|\nabla u\|_{2}^{4}-t^{4} \int_{\mathbb{R}^{3}} t^{\left(\mu_{0}-2\right) / 2} K(t x) F(u) \mathrm{d} x \\
& \leq t^{4} I(u)+\frac{a t^{2}\left(1-t^{2}\right)}{2}\|\nabla u\|_{2}^{2}<t^{4} I(u), \quad \forall u \in \mathcal{S}_{c}, c>0, t>1,
\end{aligned}
$$

where the definition of $u_{t}$ is given by (4.5). 
Let $\left\{u_{n}\right\} \subset \mathcal{S}_{c}$ be such that $I\left(u_{n}\right) \rightarrow \sigma(c)$ for any $c>0$. Since $\left\|\left(u_{n}\right)_{t}\right\|_{2}^{2}=$ $t^{4}\left\|u_{n}\right\|_{2}^{2}=t^{4} c$ for all $t>0$, it follows from (4.15) that

$$
\begin{aligned}
\sigma\left(t^{4} c\right) & \leq I\left(\left(u_{n}\right)_{t}\right) \leq t^{4} I\left(u_{n}\right)-\frac{a t^{2}\left(t^{2}-1\right)}{2}\left\|\nabla u_{n}\right\|_{2}^{2} \\
& \leq t^{4} \sigma(c)+o(1), \quad \forall t>1,
\end{aligned}
$$

which implies

$$
\sigma(t c) \leq t \sigma(c), \quad t>1, c>0 .
$$

Let $\left\{u_{n}\right\} \subset \mathcal{S}_{c}$ be such that $I\left(u_{n}\right) \rightarrow \sigma(c)$ for any $c>c^{*}$. We claim that exists a constant $\rho_{0}>0$ such that

$$
\liminf _{n \rightarrow \infty}\left\|\nabla u_{n}\right\|_{2}>\rho_{0} .
$$

Otherwise, if (4.17) is not true, then up to a subsequence, $\left\|\nabla u_{n}\right\|_{2} \rightarrow 0$, and so (4.2) yields $0>\sigma(c)=\lim _{n \rightarrow \infty} I\left(u_{n}\right)=0$. This contradiction shows that (4.17) holds. Since $\left\|\left(u_{n}\right)_{t}\right\|_{2}^{2}=t^{4}\left\|u_{n}\right\|_{2}^{2}=t^{4} c$ for all $t>0$, it follows from (4.15) and (4.17) that

$$
\begin{aligned}
\sigma\left(t^{4} c\right) & \leq I\left(\left(u_{n}\right)_{t}\right) \leq t^{4} I\left(u_{n}\right)-\frac{a t^{2}\left(t^{2}-1\right)}{2}\left\|\nabla u_{n}\right\|_{2}^{2} \\
& \leq t^{4} \sigma(c)-\frac{a t^{2}\left(t^{2}-1\right)}{2} \rho_{0}^{2}+o(1), \quad \forall t>1
\end{aligned}
$$

which implies

$$
\sigma(t c)<t \sigma(c), \quad t>1, c>c^{*} .
$$

If $\alpha>c^{*}$ and $c-\alpha>c^{*}$, we conclude from (4.18) that for any $c>c^{*}$,

$$
\sigma(c)=\frac{\alpha}{c} \sigma(c)+\frac{c-\alpha}{c} \sigma(c)<\sigma(\alpha)+\sigma(c-\alpha), \quad \forall 0<\alpha<c .
$$

If $\alpha \leq c^{*}$ or $c-\alpha \leq c^{*}$, then Lemma 4.2 (ii) implies that $\sigma(c-\alpha)=0$ or $\sigma(\alpha)=0$, and we deduce easily from (4.18) that (4.13) holds for any $c>c^{*}$. This completes the proof.

Lemma 4.4 Assume that hypotheses (K1), (K3), (F4) and (F5) hold. Then the following properties hold:

(i) $\sigma(c) \leq \sigma^{\infty}(c)$ for any $c>0$;

(ii) if (F6) holds, then $\sigma(c)$ has a minimizer for any $c>0$;

(iii) if $\left(\mathrm{F6}^{\prime}\right)$ holds, then $\sigma(c)$ has a minimizer for any $c \geq c_{*}$ and $\sigma\left(c_{*}\right)=0$.

Proof (i) Let $\left\{u_{n}\right\} \subset \mathcal{S}_{c}$ be such that $I^{\infty}\left(u_{n}\right) \rightarrow \sigma^{\infty}(c)$ for any $c>0$. Since $K_{\infty} \leq K(x)$ for all $x \in \mathbb{R}^{3}$, it follows from (1.3) and (2.7) that

$$
\sigma(c) \leq I\left(u_{n}\right) \leq I^{\infty}\left(u_{n}\right)=\sigma^{\infty}(c)+o(1)
$$

which implies that $\sigma(c) \leq \sigma^{\infty}(c)$ for any $c>0$. 
(ii) In view of Lemma 4.1 (ii), we have $\sigma(c)<0$ for any $c>0$. Let $\left\{u_{n}\right\} \subset \mathcal{S}_{c}$ be such that $I\left(u_{n}\right) \rightarrow \sigma(c)$ for any $c>0$. Then (4.2) implies that $\left\{u_{n}\right\}$ is bounded in $H^{1}\left(\mathbb{R}^{3}\right)$. Thus, we can assume that for some $\bar{u} \in H^{1}\left(\mathbb{R}^{3}\right)$ and up to a subsequence, $u_{n} \rightarrow \bar{u}$ in $H^{1}\left(\mathbb{R}^{3}\right)$. Here, we distinguish two cases: a) $\bar{u} \neq 0$ and b) $\bar{u}=0$.

Case a): $\bar{u} \neq 0$. Then $u_{n} \rightarrow \bar{u}$ in $H^{1}\left(\mathbb{R}^{3}\right), u_{n} \rightarrow \bar{u}$ in $L_{\text {loc }}^{s}\left(\mathbb{R}^{3}\right)$ for $2 \leq s<6$ and $u_{n} \rightarrow \bar{u}$ a.e. in $\mathbb{R}^{3}$. By Lemmas 3.12 and 4.3 , we have

$$
\begin{aligned}
\sigma(c) & =\lim _{n \rightarrow \infty} I\left(u_{n}\right)=I(\bar{u})+\lim _{n \rightarrow \infty}\left[I\left(u_{n}-\bar{u}\right)+\frac{b}{2}\|\nabla \bar{u}\|_{2}^{2}\left\|\nabla\left(u_{n}-\bar{u}\right)\right\|_{2}^{2}\right] \\
& \geq \sigma\left(\|\bar{u}\|_{2}^{2}\right)+\lim _{n \rightarrow \infty} \sigma\left(\left\|u_{n}-\bar{u}\right\|_{2}^{2}\right) \\
& =\sigma\left(\|\bar{u}\|_{2}^{2}\right)+\sigma\left(c-\|\bar{u}\|_{2}^{2}\right) .
\end{aligned}
$$

If $\|\bar{u}\|_{2}^{2}<c$, then relation (4.19) and Lemma 4.3 (ii) imply $\sigma(c) \geq \sigma\left(\|\bar{u}\|_{2}^{2}\right)+$ $\sigma\left(c-\|\bar{u}\|_{2}^{2}\right)>\sigma(c)$, which is impossible. This shows $\|\bar{u}\|_{2}^{2}=c=\left\|u_{n}\right\|_{2}^{2}$. Moreover, it follows from (4.19) that $u_{n} \rightarrow \bar{u}$ in $H^{1}\left(\mathbb{R}^{3}\right)$, and so $\sigma(c)=\lim _{n \rightarrow \infty} I\left(u_{n}\right)=I(\bar{u})$. Hence, $\bar{u}$ is a minimizer of $\sigma(c)$ for any $c>0$ if $\bar{u} \neq 0$.

Case b): $\bar{u}=0$, that is, $u_{n} \rightarrow 0$ in $H^{1}\left(\mathbb{R}^{3}\right)$. Then $u_{n} \rightarrow 0$ in $L_{\text {loc }}^{s}\left(\mathbb{R}^{3}\right)$ for $2 \leq s<6$ and $u_{n} \rightarrow 0$ a.e. in $\mathbb{R}^{3}$. By (K1), it is easy to check that

$$
\int_{\mathbb{R}^{3}}\left[K(x)-K_{\infty}\right] F\left(u_{n}\right) \mathrm{d} x=o(1) .
$$

Then (1.3), (2.7) and (4.20) imply

$$
I^{\infty}\left(u_{n}\right) \rightarrow \sigma(c)
$$

We claim that

$$
\delta:=\limsup _{n \rightarrow \infty} \sup _{y \in \mathbb{R}^{3}} \int_{B_{1}(y)}\left|u_{n}\right|^{2} \mathrm{~d} x>0 .
$$

In fact, if $\delta=0$, then by Lions' concentration-compactness principle [27, Lemma 1.21], one has $u_{n} \rightarrow 0$ in $L^{s}\left(\mathbb{R}^{3}\right)$ for $2<s<6$, and so (4.1) implies that $\int_{\mathbb{R}^{3}} F\left(u_{n}\right) \mathrm{d} x \rightarrow 0$. Then by (1.3), we have

$$
0>\sigma(c)=\lim _{n \rightarrow \infty} I\left(u_{n}\right)=\lim _{n \rightarrow \infty}\left(\frac{a}{2}\left\|\nabla u_{n}\right\|_{2}^{2}+\frac{b}{4}\left\|\nabla u_{n}\right\|_{2}^{4}\right) \geq 0
$$

which is impossible. It follows that $\delta>0$ and there exists $\left\{y_{n}\right\} \subset \mathbb{R}^{3}$ such that

$$
\int_{B_{1+\sqrt{3}}\left(y_{n}\right)}\left|u_{n}\right|^{2} \mathrm{~d} x \geq \frac{\delta}{2} .
$$

Let $\hat{u}_{n}(x)=u_{n}\left(x+y_{n}\right)$. Then (4.21) leads to

$$
\hat{u}_{n} \in \mathcal{S}_{c}, \quad I^{\infty}\left(\hat{u}_{n}\right) \rightarrow \sigma(c) .
$$


In view of (4.23), we may assume that there exists $\hat{u} \in H^{1}\left(\mathbb{R}^{3}\right) \backslash\{0\}$ such that, passing to a subsequence,

$$
\left\{\begin{array}{l}
\hat{u}_{n} \rightarrow \hat{u}, \quad \text { in } H^{1}\left(\mathbb{R}^{3}\right) \\
\hat{u}_{n} \rightarrow \hat{u}, \text { in } L_{\text {loc }}^{s}\left(\mathbb{R}^{3}\right), \forall s \in[1,6) \\
\hat{u}_{n} \rightarrow \hat{u}, \text { a.e. on } \mathbb{R}^{3}
\end{array}\right.
$$

Then it follows from (4.24), (4.25), (i), Lemmas 3.12 and 4.3 that

$$
\begin{aligned}
\sigma(c) & =\lim _{n \rightarrow \infty} I^{\infty}\left(\hat{u}_{n}\right) \\
& =I^{\infty}(\hat{u})+\lim _{n \rightarrow \infty}\left[I^{\infty}\left(\hat{u}_{n}-\hat{u}\right)+\frac{b}{2}\|\nabla \hat{u}\|_{2}^{2}\left\|\nabla\left(\hat{u}_{n}-\hat{u}\right)\right\|_{2}^{2}\right] \\
& \geq \sigma^{\infty}\left(\|\hat{u}\|_{2}^{2}\right)+\lim _{n \rightarrow \infty} \sigma^{\infty}\left(\left\|\hat{u}_{n}-\hat{u}\right\|_{2}^{2}\right) \\
& =\sigma^{\infty}\left(\|\hat{u}\|_{2}^{2}\right)+\sigma^{\infty}\left(c-\|\hat{u}\|_{2}^{2}\right) \geq \sigma\left(\|\hat{u}\|_{2}^{2}\right)+\sigma\left(c-\|\hat{u}\|_{2}^{2}\right),
\end{aligned}
$$

If $\|\hat{u}\|_{2}^{2}<c$, then (4.26) and Lemma 4.3 (ii) imply $\sigma(c) \geq \sigma\left(\|\hat{u}\|_{2}^{2}\right)+\sigma\left(c-\|\hat{u}\|_{2}^{2}\right)>$ $\sigma(c)$ which is impossible. Hence, $\|\hat{u}\|_{2}^{2}=c$, moreover, it follows from (4.26) that

$$
\hat{u}_{n} \rightarrow \hat{u} \text { in } H^{1}\left(\mathbb{R}^{3}\right), \quad \sigma(c) \leq I(\hat{u}) \leq I^{\infty}(\hat{u})=\lim _{n \rightarrow \infty} I^{\infty}\left(\hat{u}_{n}\right)=\sigma(c) .
$$

This shows that $\hat{u}$ is a minimizer of $\sigma(c)$ for any $c>0$.

(iii) In view of Lemma 4.1 (iii), we have $\sigma(c)<0$ for any $c>c_{*}$. Arguing as in the proof of (ii), we deduce that $\sigma(c)$ is attained for any $c>c_{*}$. Let $c_{n}=c_{*}+\frac{1}{n}$. Note that Lemma 4.2 (ii) leads to $\sigma\left(c_{n}\right)<0$ for every $n \in \mathbb{N}$. Arguing as in the proof of (ii), there exists $\left\{u_{n}\right\} \subset \mathcal{S}_{c_{n}}$ such that

$$
I\left(u_{n}\right)=\sigma\left(c_{n}\right)<0 \text { for every } n \in \mathbb{N} \text {. }
$$

By the definition of $c_{*}$ and Lemma 4.3 (i), we have $I\left(u_{n}\right)=\sigma\left(c_{n}\right) \rightarrow \sigma\left(c_{*}\right)=0$. Then (4.2) implies that $\left\{u_{n}\right\}$ is bounded in $H^{1}\left(\mathbb{R}^{3}\right)$. We then may assume that there exists $\bar{u} \in H^{1}\left(\mathbb{R}^{3}\right)$ such that, up to a subsequence, $u_{n} \rightarrow \bar{u}$ in $H^{1}\left(\mathbb{R}^{3}\right)$. To prove that $\sigma\left(c_{*}\right)$ is attained, we need to distinguish two cases: a) $\bar{u} \neq 0$ and b) $\bar{u}=0$.

Case a): $\bar{u} \neq 0$. By Lemmas 3.12, 4.2 and 4.3, we have

$$
\begin{aligned}
0 & =\sigma\left(c^{*}\right)=\lim _{n \rightarrow \infty} I\left(u_{n}\right)=I(\bar{u})+\lim _{n \rightarrow \infty}\left[I\left(u_{n}-\bar{u}\right)+\frac{b}{2}\|\nabla \bar{u}\|_{2}^{2}\left\|\nabla\left(u_{n}-\bar{u}\right)\right\|_{2}^{2}\right] \\
& \geq \sigma\left(\|\bar{u}\|_{2}^{2}\right)+\lim _{n \rightarrow \infty} \sigma\left(\left\|u_{n}-\bar{u}\right\|_{2}^{2}\right) \\
& =\sigma\left(\|\bar{u}\|_{2}^{2}\right)+\sigma\left(c^{*}-\|\bar{u}\|_{2}^{2}\right)=0
\end{aligned}
$$

which implies

$$
\lim _{n \rightarrow \infty}\left\|\nabla\left(u_{n}-\bar{u}\right)\right\|_{2}=0,
$$

and so a standard argument shows that $I(\bar{u})=\lim _{n \rightarrow \infty} I\left(u_{n}\right)=\sigma\left(c^{*}\right)=0$. To prove that $\bar{u}$ is a minimizer of $\sigma\left(c^{*}\right)$, it suffices to show that $\|\bar{u}\|_{2}^{2}=c^{*}$. By contradiction, 
let us assume that $\|\bar{u}\|_{2}^{2}<c^{*}$. Let

$$
t^{*}=\left(\frac{c^{*}}{\|\bar{u}\|_{2}^{2}}\right)^{\frac{1}{4}}
$$

Then $t^{*}>1$, and $\left\|\bar{u}_{t^{*}}\right\|_{2}^{2}=\left(t^{*}\right)^{4}\|\bar{u}\|_{2}^{2}=c^{*}$ by the scaling (4.5). Thus it follows from (4.15) that

$$
0=\sigma\left(c^{*}\right) \leq I\left(\bar{u}_{t^{*}}\right) \leq\left(t^{*}\right)^{4} I(\bar{u})+\frac{a\left(t^{*}\right)^{2}\left[1-\left(t^{*}\right)^{2}\right]}{2}\|\nabla \bar{u}\|_{2}^{2}<\left(t^{*}\right)^{4} I(\bar{u})=0 .
$$

This contradiction shows that $\|\bar{u}\|_{2}^{2}=c^{*}$. Hence, $\bar{u} \in \mathcal{S}_{c^{*}}$ and $I(\bar{u})=\sigma\left(c^{*}\right)=0$.

Case b): $\bar{u}=0$. By (2.7) and (4.20), we deduce that $I^{\infty}\left(u_{n}\right) \rightarrow 0$. We claim that (4.22) holds. In fact, if $\delta=0$, by Lions' concentration-compactness principle [27, Lemma 1.21], we obtain that $u_{n} \rightarrow 0$ in $L^{s}\left(\mathbb{R}^{3}\right)$ for $2<s<6$. By (F4) and (F6'), for any $\varepsilon>0$, there exists $C_{\varepsilon}>0$ such that

$$
|F(t)| \leq \varepsilon|t|^{\frac{10}{3}}+C_{\varepsilon}|t|^{p}, \quad \forall t \in \mathbb{R}
$$

Then (4.31) implies $\int_{\mathbb{R}^{3}} F\left(u_{n}\right) \mathrm{d} x \rightarrow 0$. Jointly with $I\left(u_{n}\right) \rightarrow 0$, we have $\left\|\nabla u_{n}\right\|_{2} \rightarrow$ 0 . From (1.3), (3.23) and (4.31), we deduce

$$
I\left(u_{n}\right) \geq \frac{1}{4}\left\|\nabla u_{n}\right\|_{2}^{2}\left(2 a+b\left\|\nabla u_{n}\right\|_{2}^{2}-4 \varepsilon\left\|u_{n}\right\|_{2}^{\frac{4}{3}}-4 C_{\varepsilon}\left\|u_{n}\right\|_{2}^{\frac{6-p}{2}}\left\|\nabla u_{n}\right\|_{2}^{\frac{3 p-10}{2}}\right), \quad \forall n \in \mathbb{N},
$$

which, together with $3 p-10>0$ and arbitrariness of $\varepsilon$, implies that $I\left(u_{n}\right) \geq 0$ for large $n \in \mathbb{N}$. This contradicts (4.28), and thus (4.22) holds. Let $\hat{u}_{n}(x)=u_{n}\left(x+y_{n}\right)$. From (4.22), there exists $\hat{u} \in H^{1}\left(\mathbb{R}^{3}\right) \backslash\{0\}$ such that, passing to a subsequence, $\hat{u}_{n} \rightarrow \hat{u}$ in $H^{1}\left(\mathbb{R}^{3}\right)$. Similarly to the proof of $(4.26)$, we have

$$
\begin{aligned}
0 & =\sigma\left(c^{*}\right)=\lim _{n \rightarrow \infty} I^{\infty}\left(\hat{u}_{n}\right) \\
& =I^{\infty}(\hat{u})+\lim _{n \rightarrow \infty}\left[I^{\infty}\left(\hat{u}_{n}-\hat{u}\right)+\frac{b}{2}\|\nabla \hat{u}\|_{2}^{2}\left\|\nabla\left(\hat{u}_{n}-\hat{u}\right)\right\|_{2}^{2}\right] \\
& \geq \sigma^{\infty}\left(\|\hat{u}\|_{2}^{2}\right)+\lim _{n \rightarrow \infty} \sigma^{\infty}\left(\left\|\hat{u}_{n}-\hat{u}\right\|_{2}^{2}\right) \\
& =\sigma^{\infty}\left(\|\hat{u}\|_{2}^{2}\right)+\sigma^{\infty}\left(c^{*}-\|\hat{u}\|_{2}^{2}\right) \geq \sigma\left(\|\hat{u}\|_{2}^{2}\right)+\sigma\left(c^{*}-\|\hat{u}\|_{2}^{2}\right)=0,
\end{aligned}
$$

which implies

$$
\lim _{n \rightarrow \infty}\left\|\nabla\left(\hat{u}_{n}-\hat{u}\right)\right\|_{2}=0 .
$$

Proceeding as in the proof of Case a), we deduce that $\hat{u} \in \mathcal{S}_{c^{*}}$ and $I(\hat{u})=\sigma\left(c^{*}\right)=0$. 


\subsection{Local Minimizers on the Constraint $\mathcal{S}_{c}$}

In this subsection, we shall look for a local minimizer of $I$ on the constraint $\mathcal{S}_{c}$, which is a critical point of $\left.I\right|_{\mathcal{S}_{c}}$.

For $k>0$, set

$$
\mathcal{S}_{c}(k):=\left\{u \in \mathcal{S}_{c}:\|\nabla u\|_{2}^{2}=k\right\}
$$

For any $0<c<c_{*}$ and $u \in \mathcal{S}_{c}$, it follows from (1.3), (3.23) and (4.31) that

$$
I(u) \geq \frac{1}{4}\|\nabla u\|_{2}^{2}\left(2 a+b\|\nabla u\|_{2}^{2}-4 \varepsilon c_{*}^{\frac{2}{3}}-4 C_{\varepsilon} c_{*}^{\frac{6-p}{4}}\|\nabla u\|_{2}^{\frac{3 p-10}{2}}\right) .
$$

Since $3 p-10>0$ and $\varepsilon$ is arbitrary, there exists $k_{0}>0$ independent of $c$ such that

$$
I^{\infty}(u) \geq I(u) \geq \frac{a k}{4}>0, \quad \forall u \in \mathcal{S}_{c}(k), 0<k \leq k_{0} .
$$

In view of Lemma 4.1 (i), we have

$$
\bar{\sigma}(c):=\inf _{u \in \mathcal{S}_{c} \backslash \bigcup_{0<k \leq k_{0}} \mathcal{S}_{c}(k)} I(u) \geq \inf _{u \in \mathcal{S}_{c}} I(u)=\sigma(c)>-\infty, \quad \forall c>0 .
$$

Lemma 4.5 Assume that hypotheses (K1), (K3), (F4), (F5) and (F6') hold. Then $\bar{\sigma}(c)$ is continuous on $(0,+\infty)$ and for any $c>0$,

$$
\bar{\sigma}(c)<\bar{\sigma}(\alpha)+\bar{\sigma}(c-\alpha), \quad \forall 0<\alpha<c .
$$

Proof Similarly to the proof of Lemma 4.3, we deduce that $\bar{\sigma}(c)$ is continuous for any $c>0$. Let $\left\{u_{n}\right\} \subset \mathcal{S}_{c} \backslash \bigcup_{0<k \leq k_{0}} \mathcal{S}_{c}(k)$ be such that $I\left(u_{n}\right) \rightarrow \bar{\sigma}(c)$ for any $c>0$. Then (4.2) implies that $\left\{u_{n}\right\}$ is bounded in $H^{1}\left(\mathbb{R}^{3}\right)$. Noting that $\left\|\nabla u_{n}\right\|_{2}^{2}>k_{0}$ and $\left\|\left(u_{n}\right)_{t}\right\|_{2}^{2}=t^{4}\left\|u_{n}\right\|_{2}^{2}=t^{4} c$ for all $n \in \mathbb{N}$ and $t>0$, it follows from (4.15) that

$$
\begin{aligned}
\bar{\sigma}\left(t^{4} c\right) & \leq I\left(\left(u_{n}\right)_{t}\right) \leq t^{4} I\left(u_{n}\right)-\frac{a t^{2}\left(t^{2}-1\right)}{2}\left\|\nabla u_{n}\right\|_{2}^{2} \\
& \leq t^{4} \bar{\sigma}(c)-\frac{a t^{2}\left(t^{2}-1\right)}{2} k_{0}^{2}+o(1), \quad \forall t>1, c>0,
\end{aligned}
$$

which implies

$$
\bar{\sigma}(t c)<t \bar{\sigma}(c), \quad t>1, c>0 .
$$

Then it follows from (4.37) that

$$
\bar{\sigma}(c)=\frac{\alpha}{c} \bar{\sigma}(c)+\frac{c-\alpha}{c} \bar{\sigma}(c)<\bar{\sigma}(\alpha)+\bar{\sigma}(c-\alpha), \quad \forall 0<\alpha<c,
$$

which completes the proof. 
Lemma 4.6 (Geometry of local minima) Assume that hypotheses (K1), (K3), (F4), (F5) and $\left(F 6^{\prime}\right)$ hold. Then there exists $0<c_{0}<c_{*}$ such that

$$
0<\bar{\sigma}(c)<\frac{a}{4} k_{0} \leq \inf _{u \in \mathcal{S}_{c}\left(k_{0}\right)} I(u), \quad \forall c \in\left(c_{0}, c_{*}\right)
$$

Proof By the definition of $c_{*}$ (see (2.2)), Lemma 4.1 (i) and (4.35), we obtain

$$
\bar{\sigma}(c) \geq \sigma(c)=0, \quad \forall 0<c<c_{*} .
$$

We first prove that $\bar{\sigma}(c)>0$ for all $0<c<c_{*}$. If not, then $\bar{\sigma}(\bar{c})=0$ for some $\bar{c} \in\left(0, c_{*}\right)$, and so there exists $\left\{u_{n}\right\} \subset \mathcal{S}_{\bar{c}} \backslash \bigcup_{0<k \leq k_{0}} \mathcal{S}_{\bar{c}}(k)$ such that $I\left(u_{n}\right) \rightarrow 0$ and $\left\|\nabla u_{n}\right\|_{2}^{2}>k_{0}$. Since $\left\|\left(u_{n}\right)_{t}\right\|_{2}^{2}=t^{4}\left\|u_{n}\right\|_{2}^{2}=t^{4} \bar{c}$ by the scaling (4.5), it follows from (4.15) that

$$
\begin{aligned}
\bar{\sigma}\left(t^{4} \bar{c}\right) & \leq \lim _{n \rightarrow \infty} I\left(\left(u_{n}\right)_{t}\right) \leq \lim _{n \rightarrow \infty}\left[t^{4} I\left(u_{n}\right)-\frac{a t^{2}\left(t^{2}-1\right)}{2}\left\|\nabla u_{n}\right\|_{2}^{2}\right] \\
& \leq-\frac{a k_{0} t^{2}\left(t^{2}-1\right)}{2}<0, \quad \forall 1<t<\left(\frac{c_{*}}{\bar{c}}\right)^{1 / 4},
\end{aligned}
$$

which contradicts (4.39). Hence, $\bar{\sigma}(c)>0$ for all $0<c<c_{*}$.

Next, inspired by [29, (3.9)], we prove that there exists $c_{0}>0$ such that the last inequality of (4.38) holds. In view of Lemma 4.4 (iii), there exists $u_{*} \in \mathcal{S}_{c_{*}}$ such that $I\left(u_{*}\right)=\sigma\left(c_{*}\right)=0$. From the continuity of $I\left(t u_{*}\right)$ on $t \in(0, \infty)$, we deduce that there exists a constant $t_{0} \in(0,1)$ sufficiently close to 1 such that

$$
I\left(t u_{*}\right)<\frac{a}{4} k_{0}, \quad \forall t_{0} \leq t \leq 1,
$$

which, together with (4.34), implies

$$
t u_{*} \in \mathcal{S}_{t c_{*}} \backslash \bigcup_{0<k \leq k_{0}} \mathcal{S}_{t c_{*}}(k), \quad \forall t_{0} \leq t \leq 1 .
$$

Letting $c_{0}=t_{0} c_{*}$, it follows from (4.34), (4.40) and (4.41) that for any $c \in\left(c_{0}, c_{*}\right)$, there exists $v_{*} \in \mathcal{S}_{c} \backslash \bigcup_{0<k \leq k_{0}} \mathcal{S}_{c}(k)$ such that

$$
\bar{\sigma}(c) \leq I\left(v_{*}\right)<\frac{a}{4} k_{0} \leq \inf _{u \in \mathcal{S}_{c}\left(k_{0}\right)} I(u)
$$

The proof is completed.

Define

$$
\bar{\sigma}^{\infty}(c):=\inf _{u \in \mathcal{S}_{c} \backslash \bigcup_{0<k \leq k_{0}} \mathcal{S}_{c}(k)} I^{\infty}(u) \geq \inf _{u \in \mathcal{S}_{c}} I^{\infty}(u)=\sigma^{\infty}(c)>-\infty, \quad \forall c>0 .
$$


Lemma 4.7 Assume that hypotheses (K1), (K3), (F4), (F5) and (F6') hold. Then

(i) $\bar{\sigma}(c) \leq \bar{\sigma}^{\infty}$ (c) for any $c>0$;

(ii) for any $c \in\left(c_{0}, c_{*}\right)$, problem (1.1) admits a solution $\left(\bar{u}_{c}, \bar{\lambda}_{c}\right) \in$ $\left(\mathcal{S}_{c} \backslash \bigcup_{0<k<k_{0}} \mathcal{S}_{c}(k)\right) \times \mathbb{R}$ such that $I\left(\bar{u}_{c}\right)=\bar{\sigma}(c)>0$.

Proof (i) Similarly to the proof of Lemma 4.4 (i), we get $\bar{\sigma}(c) \leq \bar{\sigma}^{\infty}(c)$ for any $c>0$.

(ii) By Ekeland's variational principle, there exists a sequence $\left\{u_{n}\right\} \subset \mathcal{S}_{c} \backslash$ $\bigcup_{0<k \leq k_{0}} \mathcal{S}_{c}(k)$ such that

$$
\left\{\begin{array}{l}
\bar{\sigma}(c) \leq I\left(u_{n}\right) \leq \bar{\sigma}(c)+\frac{1}{n}, \\
I(v) \geq I\left(u_{n}\right)-\frac{1}{n}\left\|u_{n}-v\right\|, \quad \forall v \in \mathcal{S}_{c} \backslash \bigcup_{0<k \leq k_{0}} \mathcal{S}_{c}(k) .
\end{array}\right.
$$

Then (4.2) implies that $\left\{u_{n}\right\}$ is bounded in $H^{1}\left(\mathbb{R}^{3}\right)$. Thus, there exists $\bar{u}_{c} \in H^{1}\left(\mathbb{R}^{3}\right)$ such that, up to a subsequence, $u_{n} \rightarrow \bar{u}_{c}$ in $H^{1}\left(\mathbb{R}^{3}\right), u_{n} \rightarrow \bar{u}_{c}$ in $L_{\text {loc }}^{s}\left(\mathbb{R}^{3}\right)$ for $2 \leq s<6$ and $u_{n} \rightarrow \bar{u}_{c}$ a.e. in $\mathbb{R}^{3}$. For brevity, we denote $\bar{u}_{c}$ by $\bar{u}$. We complete our proof in two steps as follows.

Step 1. We prove that if $\bar{u} \neq 0$, then $I(\bar{u})=\bar{\sigma}(c)$ and there exists $\bar{\lambda} \in \mathbb{R}$ such that $I^{\prime}(\bar{u})-\bar{\lambda} u=0$ for any $c \in\left(c_{0}, c_{*}\right)$.

Similarly to the proof of $[29,(3.17)]$, we have

$$
\left|\left\langle I^{\prime}\left(u_{n}\right)-\lambda_{n} u_{n}, \varphi\right\rangle\right| \leq \frac{C}{n}, \quad \forall \varphi \in \mathcal{C}_{0}^{\infty}\left(\mathbb{R}^{3}\right),
$$

where $\lambda_{n}=-\frac{\left\langle I^{\prime}\left(u_{n}\right), u_{n}\right\rangle}{c}$ and $C>0$ is a constant independent of $n$. Since $\left\{\lambda_{n}\right\}$ is bounded, we may assume that up to a subsequence, $\lambda_{n} \rightarrow \bar{\lambda}$ for some $\lambda \in \mathbb{R}$.

Now, we claim that

$$
\text { up to a subsequence, }\left\|\nabla\left(u_{n}-\bar{u}\right)\right\|_{2} \rightarrow 0 \text { if } \bar{u} \neq 0 \text {. }
$$

Assume by contradiction that $\lim _{n \rightarrow \infty}\left\|\nabla\left(u_{n}-\bar{u}\right)\right\|_{2}^{2}>0$. Then it follows from the scaling (4.5) that there exists $T_{0}>1$ such that

$$
\left\|\nabla \bar{u}_{t}\right\|_{2}^{2}=t^{2}\|\nabla \bar{u}\|_{2}^{2}>k_{0}, \quad\left\|\nabla\left(u_{n}-\bar{u}\right)_{t}\right\|_{2}^{2}=t^{2}\left\|\nabla\left(u_{n}-\bar{u}\right)\right\|_{2}^{2}>k_{0}, \quad \forall t>T_{0} .
$$

By (4.15), (4.43) and Lemma 3.12, we have

$$
\begin{aligned}
\bar{\sigma}(c)+o(1) & =I\left(u_{n}\right)=I(\bar{u})+I\left(u_{n}-\bar{u}\right)+\frac{b}{2}\|\nabla \bar{u}\|_{2}^{2}\left\|\nabla\left(u_{n}-\bar{u}\right)\right\|_{2}^{2} \\
& \geq \frac{1}{t^{4}}\left[I\left(\bar{u}_{t}\right)+I\left(\left(u_{n}-\bar{u}\right)_{t}\right)\right]+\frac{a t^{2}\left(t^{2}-1\right)}{2 t^{4}}\left[\|\nabla \bar{u}\|_{2}^{2}+\left\|\nabla\left(u_{n}-\bar{u}\right)\right\|_{2}^{2}\right] \\
& \geq \frac{1}{t^{4}}\left[\bar{\sigma}\left(t^{4}\|\bar{u}\|_{2}^{2}\right)+\bar{\sigma}\left(t^{4} c-t^{4}\|\bar{u}\|_{2}^{2}\right)\right]+\frac{a t^{2}\left(t^{2}-1\right)}{2 t^{4}}\left\|\nabla u_{n}\right\|_{2}^{2}+o(1) \\
& \geq \frac{1}{t^{4}} \bar{\sigma}\left(t^{4} c\right)+\frac{a t^{2}\left(t^{2}-1\right)}{2 t^{4}} k_{0}+o(1), \quad \forall t \geq T_{0} .
\end{aligned}
$$


From (4.2), we deduce

$$
\liminf _{t \rightarrow+\infty} \frac{\bar{\sigma}\left(t^{4} c\right)}{t^{4}} \geq 0 .
$$

By (4.48), there exists $T_{1} \geq T_{0}$ such that

$$
\frac{1}{T_{1}^{4}} \bar{\sigma}\left(T_{1}^{4} c\right) \geq-\frac{a k_{0}}{16}, \quad \frac{a T_{1}^{2}\left(T_{1}^{2}-1\right)}{2 t^{4}} k_{0} \geq \frac{3 a k_{0}}{8} .
$$

Then (4.38), (4.47) and (4.49) lead to

$$
\frac{a k_{0}}{4}>\bar{\sigma}(c) \geq \frac{1}{T_{1}^{4}} \bar{\sigma}\left(T_{1}^{4} c\right)+\frac{a T_{1}^{2}\left(T_{1}^{2}-1\right)}{2 t^{4}} k_{0} \geq \frac{5 a k_{0}}{16}
$$

This contradiction shows that (4.45) holds. Thus, from (F4), (F5), (4.43), (4.44) and (4.45), we derive

$$
\bar{\sigma}(c)=\lim _{n \rightarrow \infty} I\left(u_{n}\right)=I(\bar{u})
$$

and

$$
\begin{aligned}
0= & \lim _{n \rightarrow \infty}\left\langle I^{\prime}\left(u_{n}\right)-\lambda_{n} u_{n}, \varphi\right\rangle \\
= & \lim _{n \rightarrow \infty}\left[a \int_{\mathbb{R}^{3}} \nabla u_{n} \cdot \nabla \varphi \mathrm{d} x+b\left\|\nabla u_{n}\right\|_{2}^{2} \int_{\mathbb{R}^{3}} \nabla u_{n} \cdot \nabla \varphi \mathrm{d} x\right. \\
& \left.-\int_{\mathbb{R}^{3}} K(x) f\left(u_{n}\right) \varphi \mathrm{d} x-\lambda_{n} \int_{\mathbb{R}^{3}} u_{n} \varphi \mathrm{d} x\right] \\
= & a \int_{\mathbb{R}^{3}} \nabla \bar{u} \cdot \nabla \varphi \mathrm{d} x+b\|\nabla \bar{u}\|_{2}^{2} \int_{\mathbb{R}^{3}} \nabla \bar{u} \cdot \nabla \varphi \mathrm{d} x-\int_{\mathbb{R}^{3}} K(x) f(\bar{u}) \varphi \mathrm{d} x-\bar{\lambda} \int_{\mathbb{R}^{3}} \bar{u} \varphi \mathrm{d} x \\
= & \left\langle I^{\prime}(\bar{u})-\bar{\lambda} \bar{u}, \varphi\right\rangle, \quad \forall \varphi \in \mathcal{C}_{0}^{\infty}\left(\mathbb{R}^{3}\right) .
\end{aligned}
$$

By Fatou's lemma, we have $\|\bar{u}\|_{2}^{2} \leq \liminf _{n \rightarrow \infty}\left\|u_{n}\right\|_{2}^{2}=c$. In view of (4.51) and (4.52), to finish the proof of Step 1 , it suffices to show that $\|\bar{u}\|_{2}^{2}=c$.

Suppose by contradiction that $\|\bar{u}\|_{2}^{2}<c$. As in [29, (3.14)], for any $\varepsilon>0$, there exists $\delta=\delta(\varepsilon)>0$ such that if $u \in \bigcup_{k_{0}-\delta \leq k \leq k_{0}+\delta} \mathcal{S}_{c}(k)$, then

$$
I(u) \geq \inf _{u \in \mathcal{S}_{c}\left(k_{0}\right)} I(u)-\varepsilon,
$$

and so

$$
u_{n} \in \mathcal{S}_{c} \backslash \bigcup_{0 \leq k \leq k_{0}+\delta} \mathcal{S}_{c}(k), \text { for } n \text { large enough, }
$$

that is, $\left\{u_{n}\right\}$ stays away from the boundary. Jointly with (4.45), we have $\|\nabla \bar{u}\|_{2}^{2}>$ $k_{0}$. Let $\bar{t}:=\left(c /\|\bar{u}\|_{2}^{2}\right)^{1 / 4}>1$. By the scaling (4.5), one has $\left\|\bar{u}_{\bar{t}}\right\|_{2}^{2}=\bar{t}^{4}\|\bar{u}\|_{2}^{2}$ and $\left\|\nabla \bar{u}_{\bar{t}}\right\|_{2}^{2}=\bar{t}^{2}\|\nabla \bar{u}\|_{2}^{2}>k_{0}$. Then it follows from (4.15) and (4.51) that 


$$
\begin{aligned}
\bar{t}^{4} \bar{\sigma}(c) & =\bar{t}^{4} I(\bar{u}) \geq I\left(\bar{u}_{\bar{t}}\right)+\frac{a \bar{t}^{2}\left(\bar{t}^{2}-1\right)}{2}\|\nabla \bar{u}\|_{2}^{2} \\
& \geq \bar{\sigma}(c)+\frac{a \bar{t}^{2}\left(\bar{t}^{2}-1\right)}{2} k_{0},
\end{aligned}
$$

which implies

$$
\bar{\sigma}(c) \geq \frac{a \sqrt{c} k_{0}}{2(\|\bar{u}\|+\sqrt{c})}>\frac{a k_{0}}{4}, \quad \forall c \in\left(c_{0}, c_{*}\right) .
$$

This contradicts (4.38). Hence, we have $\|\bar{u}\|_{2}^{2}=c$. Moreover, (4.52) implies that $(\bar{u}, \bar{\lambda}) \in\left(\mathcal{S}_{c} \backslash \bigcup_{0<k \leq k_{0}} \mathcal{S}_{c}(k)\right) \times \mathbb{R}$ solves problem (1.1). The proof of Step 1 is completed.

Step 2. We prove that $\bar{u} \neq 0$.

Assume by contradiction that $\bar{u}=0$, that is, $u_{n} \rightarrow 0$ in $H^{1}\left(\mathbb{R}^{3}\right)$. Then $u_{n} \rightarrow 0$ in $L_{\text {loc }}^{s}\left(\mathbb{R}^{3}\right)$ for $2 \leq s<6$ and $u_{n} \rightarrow 0$ a.e. in $\mathbb{R}^{3}$. Then (1.3), (2.7), (4.20) and (4.43) imply

$$
I^{\infty}\left(u_{n}\right) \rightarrow \bar{\sigma}(c) .
$$

We first prove that $\left\{u_{n}\right\}$ is not vanishing, that is, relation (4.22) holds. Otherwise, if $\left\{u_{n}\right\}$ is vanishing, by Lions' concentration-compactness principle [27, Lemma 1.21], one has $u_{n} \rightarrow 0$ in $L^{s}\left(\mathbb{R}^{3}\right)$ for $2<s<6$, and so (4.31) implies that $\int_{\mathbb{R}^{3}} F\left(u_{n}\right) \mathrm{d} x \rightarrow 0$. Since $\left\{u_{n}\right\} \subset \mathcal{S}_{c} \backslash \bigcup_{0<k \leq k_{0}} \mathcal{S}_{c}(k)$, by (1.3), (4.38) and (4.43), we have

$$
\begin{aligned}
\frac{a k_{0}}{2}+\frac{b k_{0}^{2}}{4} & \leq \lim _{n \rightarrow \infty}\left(\frac{a}{2}\left\|\nabla u_{n}\right\|_{2}^{2}+\frac{b}{4}\left\|\nabla u_{n}\right\|_{2}^{4}\right) \\
& =\lim _{n \rightarrow \infty} I\left(u_{n}\right)=\bar{\sigma}(c)<\frac{a k_{0}}{4},
\end{aligned}
$$

which is impossible. Hence, we have (4.22) holds, and so there exists $\left\{y_{n}\right\} \subset \mathbb{R}^{3}$ such that (4.23) holds. Let $\tilde{u}_{n}(x)=u_{n}\left(x+y_{n}\right)$. Then (4.55) leads to

$$
\tilde{u}_{n} \in \mathcal{S}_{c} \backslash \bigcup_{0<k \leq k_{0}} \mathcal{S}_{c}(k), \quad I^{\infty}\left(\tilde{u}_{n}\right) \rightarrow \bar{\sigma}(c) .
$$

We may assume that there exists $\tilde{u} \in H^{1}\left(\mathbb{R}^{3}\right) \backslash\{0\}$ such that, passing to a subsequence,

$$
\left\{\begin{array}{l}
\tilde{u}_{n} \rightarrow \tilde{u}, \quad \text { in } H^{1}\left(\mathbb{R}^{3}\right) \\
\tilde{u}_{n} \rightarrow \tilde{u}, \text { in } L_{\text {loc }}^{s}\left(\mathbb{R}^{3}\right), \forall s \in[1,6) \\
\tilde{u}_{n} \rightarrow \tilde{u}, \text { a.e. on } \mathbb{R}^{3}
\end{array}\right.
$$

Similarly to the proof of [29, (3.17)], we have

$$
\left|\left\langle\left(I^{\infty}\right)^{\prime}\left(\tilde{u}_{n}\right)-\lambda_{n} \tilde{u}_{n}, \varphi\right\rangle\right| \leq \frac{C}{n}, \quad \forall \varphi \in \mathcal{C}_{0}^{\infty}\left(\mathbb{R}^{3}\right),
$$

where $\lambda_{n}$ and $C>0$ are the same as that of (4.44). Arguing as in (4.45), (4.51) and (4.52), we prove that 


$$
\text { up to a subsequence }\left\|\nabla\left(\tilde{u}_{n}-\tilde{u}\right)\right\|_{2} \rightarrow 0
$$

and

$$
\bar{\sigma}(c)=\lim _{n \rightarrow \infty} I^{\infty}\left(\tilde{u}_{n}\right)=I^{\infty}(\tilde{u}) .
$$

Moreover, as in the proof of Step 1 , we have $\|\tilde{u}\|_{2}^{2}=c$ and $\|\nabla \tilde{u}\|_{2}^{2}>k_{0}$. Hence, $\tilde{u} \in \mathcal{S}_{c} \backslash \bigcup_{0<k \leq k_{0}} \mathcal{S}_{c}(k)$. Since $K(x) \geq(\not \equiv) K_{\infty}$ and $\|\tilde{u}\|_{2}^{2}=c>0$, it follows that there exist $r>0$ and $\bar{x}_{1}, \bar{x}_{2} \in \mathbb{R}^{3}$ such that

$$
K(x)-K_{\infty}>0, \quad \forall\left|x-\bar{x}_{1}\right|<r, \quad|\tilde{u}(x)|>0, \quad \forall\left|x-\bar{x}_{2}\right|<r .
$$

Let $\hat{u}(x)=\tilde{u}\left(x-\bar{x}_{1}+\bar{x}_{2}\right)$. Then $\|\hat{u}\|_{2}^{2}=c,\|\nabla \hat{u}\|_{2}^{2}>k_{0}$ and $I^{\infty}(\hat{u})=I^{\infty}(\tilde{u})$, which together with (1.3), (2.7), implies that

$$
\bar{\sigma}(c)=I^{\infty}(\hat{u})>I(\hat{u}) \geq \bar{\sigma}(c) .
$$

This contradiction shows that $\bar{u} \neq 0$.

Proof of Theorem 2.2 Note that if $u_{c}$ is a critical point of $\left.I\right|_{\mathcal{S}_{c}}$, then there exists $\lambda_{c} \in \mathbb{R}$ such that $I^{\prime}\left(u_{c}\right)-\lambda_{c} u_{c}=0$. Combining Lemma 4.2, 4.4 and 4.7, we conclude the proof of Theorem 2.2.

Acknowledgements This work was partially supported by the National Natural Science Foundation of China (No: 11571370). The authors thank the anonymous referee for the careful reading of this paper and for several suggestions which improved the initial version of the present work.

Open Access This article is licensed under a Creative Commons Attribution 4.0 International License, which permits use, sharing, adaptation, distribution and reproduction in any medium or format, as long as you give appropriate credit to the original author(s) and the source, provide a link to the Creative Commons licence, and indicate if changes were made. The images or other third party material in this article are included in the article's Creative Commons licence, unless indicated otherwise in a credit line to the material. If material is not included in the article's Creative Commons licence and your intended use is not permitted by statutory regulation or exceeds the permitted use, you will need to obtain permission directly from the copyright holder. To view a copy of this licence, visit http://creativecommons.org/licenses/by/4.0/.

\section{References}

1. Arosio, A., Panizzi, S.: On the well-posedness of the Kirchhoff string. Trans. Am. Math. Soc. 348, 305-330 (1996)

2. Azzollini, A.: A note on the elliptic Kirchhoff equation in $\mathbb{R}^{N}$ perturbed by a local nonlinearity. Commun. Contemp. Math. 17, 1450039 (2015)

3. Bellazzini, J., Siciliano, G.: Scaling properties of functionals and existence of constrained minimizers. J. Funct. Anal. 261, 2486-2507 (2011)

4. Bellazzini, J., Jeanjean, L., Luo, T.: Existence and instability of standing waves with prescribed norm for a class of Schrödinger-Poisson equations. Proc. Lond. Math. Soc. 107, 303-339 (2013)

5. Bernstein, S.: Sur une class d'équations fonctionnelles aux dérivées partielles. Bull. Acad. Sci. URSS. Sér. Math. 4, 17-26 (1940)

6. Cavalcanti, M.M., Cavalcanti, V.N.D., Soriano, J.A.: Global existence and uniform decay rates for the Kirchhoff-Carrier equation with nonlinear dissipation. Adv. Differ. Equ. 6, 701-730 (2001)

7. Chen, J.S., Li, L.: Multiple solutions for the nonhomogeneous Kirchhoff equations on $\mathbb{R}^{3}$. Nonlinear Anal. Real World Appl. 14, 1477-1486 (2013) 
8. Chen, S.T., Tang, X.H.: Berestycki-Lions conditions on ground state solutions for a nonlinear Schrödinger equation with variable potentials. Adv. Nonlinear Anal. 9, 496-515 (2020)

9. Chen, S.T., Tang, X.H.: On the planar Schrödinger-Poisson system with the axially symmetric potential. J. Differ. Equ. 268, 945-976 (2020)

10. Chen, S.T., Fiscella, A., Pucci, P., Tang, X.H.: Semiclassical ground state solutions for critical Schrödinger-Poisson systems with lower perturbations. J. Differ. Equ. 268, 2672-2716 (2020)

11. Cingolani, S., Jeanjean, L.: Stationary waves with prescribed $L^{2}$-norm for the planar SchrödingerPoisson system. SIAM J. Math. Anal. 51, 3533-3568 (2019)

12. D'Ancona, P., Spagnolo, S.: Global solvability for the degenerate Kirchhoff equation with real analytic data. Invent. Math. 108, 247-262 (1992)

13. Deng, Y.B., Peng, S.J., Shuai, W.: Existence and asymptotic behavior of nodal solutions for the Kirchhoff-type problems in $\mathbb{R}^{3}$. J. Funct. Anal. 269, 3500-3527 (2015)

14. Jeanjean, L.: Existence of solutions with prescribed norm for semilinear elliptic equations. Nonlinear Anal. 28, 1633-1659 (1997)

15. Jeanjean, L., Luo, T.: Sharp nonexistence results of prescribed $L^{2}$-norm solutions for some class of Schrödinger-Poisson and quasi-linear equations. Z. Angew. Math. Phys. 64, 937-954 (2013)

16. Kirchhoff, G.: Mechanik. Teubner, Leipzig (1883)

17. Li, G.B., Ye, H.Y.: Existence of positive ground state solutions for the nonlinear Kirchhoff type equations in $\mathbb{R}^{3}$. J. Differ. Equ. 257, 566-600 (2014)

18. Li, G.B., Ye, H.Y.: Existence of positive solutions for nonlinear Kirchhoff type problems in $\mathbb{R}^{3}$ with critical Sobolev exponent. Math. Methods Appl. Sci. 37, 2570-2584 (2014)

19. Lions, J.L.: On some questions in boundary value problems of mathematical physics. North-Holl. Mathe. Stud. 30, 284-346 (1978)

20. Mingqi, X., Rădulescu, V.D., Zhang, B.L.: Nonlocal Kirchhoff diffusion problems: local existence and blow-up of solutions. Nonlinearity 31, 3228-3250 (2018)

21. Mingqi, X., Rădulescu, V.D., Zhang, B.L.: Fractional Kirchhoff problems with critical TrudingerMoser nonlinearity. Calc. Var. Partial Differ. Equ. 58(2), 27 (2019)

22. Molica Bisci, G., Rădulescu, V.D., Servadei, R.: Variational Methods for Nonlocal Fractional Problems. Encyclopedia of Mathematics and its Applications, vol. 162. Cambridge University Press, Cambridge (2016)

23. Papageorgiou, N.S., Rădulescu, V.D., Repov̌̌, D.D.: Nonlinear Analysis-Theory and Methods. Springer Monographs in Mathematics. Springer, Cham (2019)

24. Tang, X.H., Chen, S.T.: Ground state solutions of Nehari-Pohožaev type for Kirchhoff-type problems with general potentials. Calc. Var. Partial Differ. Equ. 56, 110-134 (2017)

25. Tang, X.H., Chen, S.T.: Singularly perturbed Choquard equations with nonlinearity satisfying Berestycki-Lions assumptions. Adv. Nonlinear Anal. 9, 413-437 (2020)

26. Tolksdorf, P.: Regularity for some general class of quasilinear elliptic equations. J. Differ. Equ. 51, 126-150 (1984)

27. Willem, M.: Minimax Theorems. Progress in Nonlinear Differential Equations and their Applications, vol. 24. Birkhäuser Boston Inc., Boston (1996)

28. Xie, W., Chen, H.: Existence and multiplicity of normalized solutions for nonlinear Kirchhoff-type problems. Comput. Math. Appl. 76, 579-591 (2018)

29. Ye, H.Y.: The sharp existence of constrained minimizers for a class of nonlinear Kirchhoff equations. Math. Methods Appl. Sci. 38, 2663-2679 (2015)

30. Ye, H.Y.: The existence of normalized solutions for $L^{2}$-critical constrained problems related to Kirchhoff equations. Z. Angew. Math. Phys. 66, 1483-1497 (2015)

31. Ye, H.Y.: The mass concentration phenomenon for $L^{2}$-critical constrained problems related to Kirchhoff equations. Angew. Math. Phys. (2016). https://doi.org/10.1007/s00033-016-0624-4

32. Ye, H.Y.: The existence of least energy nodal solutions for some class of Kirchhoff equations and Choquard equations in $\mathbb{R}^{N}$. J. Math. Anal. Appl. 431, 935-954 (2015)

33. Zeng, X., Zhang, Y.: Existence and uniqueness of normalized solutions for the Kirchhoff equation. Appl. Math. Lett. 74, 52-59 (2017)

Publisher's Note Springer Nature remains neutral with regard to jurisdictional claims in published maps and institutional affiliations. 